\title{
A GENEALOGY FOR FINITE KNEADING SEQUENCES OF BIMODAL MAPS ON THE INTERVAL
}

\author{
JOHN RINGLAND AND CHARLES TRESSER
}

\begin{abstract}
We generate all the finite kneading sequences of one of the two kinds of bimodal map on the interval, building each sequence uniquely from a pair of shorter ones. There is a single pair at generation 0 , with members of length 1. Concomitant with this genealogy of kneading sequences is a unified genealogy of all the periodic orbits. (See Figure 0.)
\end{abstract}

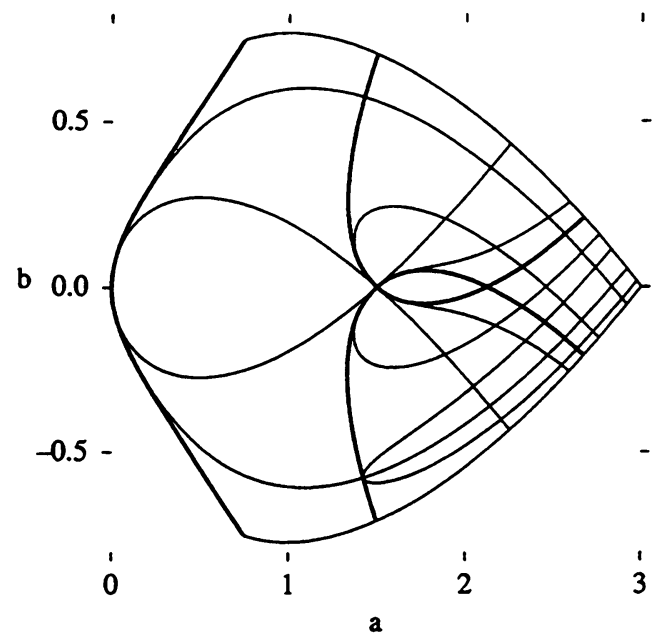

FIGURE 0 . Loci of some finite kneading sequences for a two-parameter cubic family

\section{INTRODUCTION}

A continuous map $f:[0,1] \rightarrow[0,1]$ is $\{+-+\}$-bimodal if there exist two points $c, k$ with $0<c<k<1$ such that $f$ is increasing on $[0, c] \cup[k, 1]$, and decreasing on $[c, k]$. If $f$ is constant in some interval containing $c, c$ is chosen as the middle of this interval, and the same convention holds for $k$. The points $c$ and $k$ are called the turning points of the map.

Following [MT], we code the dynamics of such a map in the following way. Given a $\{+-+\}$-bimodal map, we define:

Received by the editors May 17, 1993.

1991 Mathematics Subject Classification. Primary 58F03, 28D20, 58F14, 26 A 18. 
- the address $a(x)$ of $x \in[0,1]$ as

$L$ if $x \in[0, c)$,

$C$ if $x=c$,

$M$ if $x \in(c, k)$,

$K$ if $x=k$,

$R$ if $x \in(k, 1]$,

- the extended itinerary of $x$ as the word

$$
I_{e}(x)=a(x) a(f(x)) a\left(f^{2}(x)\right) \ldots
$$

in the alphabet $\mathscr{A}=\{L, C, M, K, R\}$, where

$$
f^{0}=\mathrm{Id}, \quad f^{n}=f \circ f^{n-1} \text { for } n>0,
$$

- the itinerary of $x$ as the word $I_{e}(x)$ if it does not contain any $C$ or $K$, and as the finite word obtained from $I_{e}(x)$ by cutting after the first symbol $C$ or $K$ if any,

- and the kneading data of the map $f$ as the pair

$$
K(f)=\left(K^{+}(f), K^{-}(f)\right)=(I(f(c)), I(f(k))),
$$

where the special itineraries $K^{+}(f)$ and $K^{-}(f)$ are called the kneading sequences of $f$.

What we do here is generate hierarchically all the finite kneading sequences realizable by $\{+-+\}$-bimodal maps in a natural way that (i) is related to local bifurcation (Theorems 1 and 2), (ii) allows for a direct comparison of entropies (Theorem 3), and (iii) provides a way to link different dynamical behavior (Theorem 4). Our result can be compared to the generation of all itineraries for rotations using the Farey tree (see, e.g., [STZ] and references therein to an abundant previous literature on this related subject) or the decomposition of kneading sequences for Lorenz-like maps [TW]. It is also to be compared with a previous genealogical result for unimodal maps [D].

The paper is organized as follows. In $\S I I$, we present a symbolic space, $\mathscr{V}$, directly motivated by the kneading theory of $\{+-+\}$-bimodal maps, whose finite words are all possible finite kneading sequences of such maps, and we formulate our main results about the space $\mathscr{V}$. Proofs are provided in $\S I I I$. The main result in $\S I I$ can be interpreted as a hierarchical organization of all the finite kneading sequences of $\{+-+\}$-bimodal maps, giving in particular a new partial order on these sequences: this will be discussed in $\S I V$ in connection with what we call the topological entropy of a kneading sequence. In $\S \mathrm{V}$, we use stunted $\{+-+\}$-saw-tooth maps to display these results graphically: this two-parameter family is simple enough to allow for a fairly complete description of the subset $\mathbf{S}$ of the parameter space corresponding to maps with (at least) one finite kneading sequence. Hence one can draw pictures which are provably topologically exact. Taking some quotient of the structure so generated for the stunted $\{+-+\}$-sawtooth maps yields the conjectural picture for the counterpart to $\mathbf{S}$ in families of smooth maps, proposed in [RS1] and [RS2] on the basis of local bifurcation diagrams. A small subset of the conjectural picture, computed with an explicit family of cubic maps, is presented in Figure 0 for orientation. (More fully described figures appear in the last section.) Early related pictures appeared in [FK] and [GP]: we refer the reader to [MaT1-MaT3], [RS1], [RS2] and the more recent paper [Mil] for more complete references. 
The $\{+-+\}$-bimodal maps are often studied because they serve to describe the dynamics of the simplest nonmonotonic degree-one circle maps (see, e.g., [B] and [MaT1]). There is another kind of bimodal map, the $\{-+-\}$ bimodal maps, which are decreasing on the first interval of monotonicity. The $\{-+-\}$-bimodal maps have a somewhat more complicated bifurcation diagram, as suggested by some partial results in [MaT2, MaT3], and a description of this class of maps in parallel to what is done here will be offered in a forthcoming paper $[R]$.

\section{STATEMENT OF THE SYMBOLIC RESUlTS}

Sections II and III constitute a self-contained symbolic discussion, and do not rely on any properties of interval maps. However, the theorems developed in these sections will later be interpreted as providing a genealogy for the kneading sequences of $\{+-+\}$-bimodal maps, so we do mention, as we go, some motivations in the theory of such maps.

II.1. Preliminary definitions. We first make enough definitions to allow us to state the main results. Following standard usage, we define $\mathscr{A}^{*}$ to be the set of empty or finite words written with alphabet $\mathscr{A}=\{L, C, M, K, R\}$, and $\mathscr{A}$ to be the set of infinite words written with alphabet $\mathscr{A}$. We further define $\mathscr{W}$ to be the set of all words in $\mathscr{A}$ truncated after the first $C$ or $K$ if any, and $\mathscr{W}^{\#}$ to be the set of finite words in $\mathscr{W}$. We remark that $\mathscr{W}$ contains all kneading sequences of bimodal maps, and $\mathscr{W}^{\#}$ all the finite kneading sequences. In order to discuss subwords of words in $\mathscr{W}^{\#}$ we introduce the reduced alphabet $\mathscr{B}=\{L, M, R\}$ and define $\mathscr{B}^{*}$ as the set of empty or finite words written with alphabet $\mathscr{B}$. Note that each word in $\mathscr{W}^{\#}$ is of the form $B Q$ with $B \in \mathscr{B}^{*}$ and $Q \in\{C, K\}$.

In what follows, we will use upper case letters not in $\mathscr{A}$ to denote words in the various sets defined above. For $F \in \mathscr{A}^{*}, G \in \mathscr{A}^{*} \cup \mathscr{A}$, we write $F G$ to

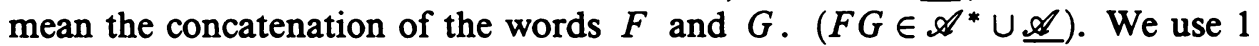
to denote the empty word: for $G \in \mathscr{A}^{*} \cup \underline{\mathscr{A}}, 1 G=G 1=G$. For $F \in \mathscr{A}^{*}$, we use $[F]^{n}$ to mean the concatenation of $n$ copies of $F:[F]^{0}=1$, and for $n>0,[F]^{n}=F[F]^{n-1} .|W|$ stands for the length of (number of letters in) $W \in \mathscr{A}^{*}$.

We define an ordering $\leq$ on $\mathscr{W}$ by first imposing the following ordering on $\mathscr{A}: L<C<M<K<R$. To induce an ordering on $\mathscr{W}$, we define the parity of a word $W$ in $\mathscr{B}^{*}$ as even if it contains an even number of $M$ 's, and odd otherwise, and a parity function $\rho: \mathscr{B}^{*} \rightarrow\{-1,1\}$ by

$$
\rho(W)= \begin{cases}+1 & \text { if } W \text { is even }, \\ -1 & \text { if } W \text { is odd } .\end{cases}
$$

Then, if two distinct words $V$ and $W$ in $\mathscr{W}$ are written as $V=T A X$ and $W=T B Y$, with $T \in \mathscr{B}^{*}, A$ and $B \in \mathscr{A}$ and $A<B$, and $X$ and $Y \in \mathscr{W}$,

$$
V<W \quad \text { if } \rho(T)=+1, \quad W<V \quad \text { if } \rho(T)=-1 .
$$

We remark that for any $\{+-+\}$-bimodal map on $[0,1]$ and any $x, y$ in $[0,1]$, we have $x<y \Rightarrow I(x) \leq I(y)$ and $I(x)<I(y) \Rightarrow x<y$ (see, e.g., $[\mathrm{MT}])$.

In order to specify the words in $\mathscr{W}$ that are realizable as the kneading sequence of a bimodal map, we introduce the shift map $\sigma: \mathscr{A}^{*} \cup \underline{\mathscr{A}} \rightarrow \mathscr{A}^{*} \cup \underline{\mathscr{A}}$, 
defined by: $\sigma(W)=1$ if $|W| \leq 1$, and, for $|W|>1$, writing $W=A W^{\prime}$, with $|A|=1, \sigma(W)=W^{\prime}$. We will use the notation $\sigma^{0}(W)=W$, and $\sigma^{k}(W)=$ $\sigma\left(\sigma^{k-1}(W)\right), k>0$. A word $W$ in $\mathscr{W}$ will be called minimal if it bounds all its shifts from below $\left(W \leq \sigma^{k}(W) \forall k \in\{0,1, \ldots,|W|-1\}\right.$ ), maximal if it bounds all its shifts from above $\left(\sigma^{k}(W) \leq W \forall k \in\{0,1, \ldots,|W|-1\}\right)$, and extremal if it is minimal or maximal. We remark that being extremal is a necessary and sufficient condition that the word occur as a kneading sequence of some $\{+-+\}$-bimodal map: necessity is rather obvious, and a proof of sufficiency is easily provided using stunted saw-tooth families. We are thus motivated to define $\mathscr{V}$ as the set of all extremal words in $\mathscr{V}$, and $\mathscr{V}^{\#}$ as the set of finite words in $\mathscr{V}$.

A pair, $(V, W)$, of words in $\mathscr{V}$ will be called admissible iff all shifts of $V$ and $W$ are bounded from above by $V$ and from below by $W$ : that is, iff $\forall i \in\{0,1, \ldots,|W|-1\}, W \leq \sigma^{i}(W)<V$ and $\forall j \in\{0,1, \ldots,|V|-1\}$, $W<\sigma^{j}(V) \leq V$. As motivation we point out that admissibility is a necessary and sufficient condition that the pair appear as the kneading data of some $\{+-+\}$-bimodal map.

In the following, we will frequently be concerned with the replacement in a word of $C$ by $L$ or $M$, and of $K$ by $M$ or $R$. We define $C^{-1}=L, C^{+1}=$ $M, K^{-1}=M, K^{+1}=R$. Note that $C^{-1}<C<C^{+1}$ and $K^{-1}<K<K^{+1}$. This exponential notation will frequently be used in conjunction with the parity function. Observe that $A C^{-\rho(A)}<A C<A C^{+\rho(A)}$ and $A K^{-\rho(A)}<A K<$ $A K^{+\rho(A)}$.

\section{II.2. Hierarchical generation of all words in $\mathscr{V}^{\#}$.}

Definitions. Among finite admissible pairs we single out three classes.

An admissible pair of words in $\mathscr{V}^{\#} \times \mathscr{V}^{\#}$ of the form $(A Q, B Q), Q \in$ $\{C, K\}$, will be called a $\psi$-seed.

An admissible pair of words in $\mathscr{V}^{\#} \times \mathscr{V}^{\#}$ of the form $(A K, B C)$ will be called a $\chi$-seed.

In the sequel, $\mathscr{V}^{\#} \times \mathscr{V}^{\#}$ will be understood as the quotient of the bare product by the relation $(C, C) \approx(K, K) ;(C, C)$ and $(K, K)$ will be considered as two equivalent representations of the $\alpha$-seed.

Remarks. The $\psi$-seed corresponds to the kneading data of a map with one critical point periodic and in the orbit of the other. The $\chi$-seed corresponds to the kneading data of a map with both critical points belonging to the same periodic orbit. The $\alpha$-seed is thought of as the kneading data of a map with a 1-periodic cubic-like inflection point. The class of finite admissible pairs not mentioned above are those of the form $(A C, B K)$, which correspond to the kneading data of a map whose critical points belong to two different periodic orbits. They are not relevant to our present purposes, for they do not participate in the hierarchy: $A C$ and $B K$ are the only finite kneading sequences that exist for smooth maps arbitrarily close to one with kneading data $(A C, B K)$.

\section{Theorem 1.}

$\alpha$. The words

$$
\alpha_{+n} \equiv[R]^{n-1} K, \quad n \in\{1,2, \ldots\}
$$


are maximal, and the words

$$
\alpha_{-n} \equiv[L]^{n-1} C, \quad n \in\{1,2, \ldots\},
$$

are minimal. We say that these words emanate from the $\alpha$-seed.

$\psi$. If $(A K, B K)$ is a $\psi$-seed, then the words

$$
\Psi_{ \pm n}(A K, B K) \equiv A K^{ \pm \rho(A)}\left[B K^{\rho(B)}\right]^{n-1} B K, \quad n \in\{1,2, \ldots\},
$$

are maximal.

If $(A C, B C)$ is a $\psi$-seed, then the words

$$
\Psi_{ \pm n}(A C, B C) \equiv B C^{ \pm \rho(B)}\left[A C^{-\rho(A)}\right]^{n-1} A C, \quad n \in\{1,2, \ldots\},
$$

are minimal. We say that the words $\Psi_{ \pm n}(A Q, B Q)$ emanate from the $\psi$-seed $(A Q, B Q)$.

$\chi$.If $(A K, B C)$ is a $\chi$-seed, then the words

$$
\begin{aligned}
& \beta_{(+1, \pm 1)}(A K, B C) \equiv A K^{ \pm \rho(A)} B C, \\
& \chi_{(+1, \pm n)}(A K, B C) \equiv A K^{+\rho(A)}\left[B C^{ \pm \rho(B)} A K^{- \pm \rho(A)}\right]^{n-1} B C^{-\rho(B)} A K, \\
& n \in\{1,2, \ldots\},
\end{aligned}
$$

are maximal, and the words

$$
\begin{aligned}
& \beta_{(-1, \pm 1)}(A K, B C) \equiv B C^{ \pm \rho(B)} A K, \\
& \chi_{(-1, \pm n)}(A K, B C) \equiv B C^{-\rho(B)}\left[A K^{- \pm \rho(A)} B C^{ \pm \rho(B)}\right]^{n-1} A K^{-( \pm \rho(A))} B C, \\
& n \in\{1,2, \ldots\},
\end{aligned}
$$

are minimal. We say that these words emanate from the $\chi$-seed $(A K, B C)$.

Remark. The word emanation is used because in smooth bimodal families an emanating word exists as a kneading sequence of the map on a curve in the two-dimensional parameter space that abuts on the point where the seed from which the sequence emanates is the kneading data [RS2].

We now state the main result of the paper.

Theorem 2. Every word in $\mathscr{V}^{\#}$ emanates from a unique seed.

\section{Proof of Theorems 1 and 2}

Theorems 1 and 2 are proved using a substantial number of preparatory lemmas. The proofs of these lemmas are rather mechanical, and involve much near-repetition. For this reason, we state most of them without proof. Exceptions are Lemma B which is fundamental, being used in the proof of nearly every other statement, and Lemma C3a whose proof is included to illustrate the character of the other proofs. The proofs of Theorems 1 and 2, using the lemmas, are given in full.

In addition to the terminology introduced in §II, we will use the following. We define the truncations, $\tau_{k}(W)$, of a word $W$ in $\mathscr{A}^{*} \cup \mathscr{A}, k$ in $\{0,1, \ldots\}$, by $W=\tau_{k}(W) \sigma^{k}(W)$. Note that $\tau_{0}(W)=1$, and $\tau_{k}(W)=W$ for $k>|W|$. Thus $\sigma^{k}(W)$ is the word obtained from $W$ by deleting its first $k$ letters, and $\tau_{k}(W)$ is the word consisting of the first $k$ letters of $W$. Note that for all $k$ 
in $\{0, \ldots,|W|\},\left|\sigma^{k}(W)\right|=|W|-k$, and $\left|\tau_{k}(W)\right|=k$. We define an order function, $O: \mathscr{W} \times \mathscr{W} \rightarrow\{-1,0,1\}$, by

$$
O(V, W)= \begin{cases}+1 & \text { if } V<W \\ 0 & \text { if } V=W \\ -1 & \text { if } W<V .\end{cases}
$$

Note that for all $(V, W)$ in $\mathscr{V}^{\#} \times V^{\#}, O(W, V)=-O(V, W)$, and if $\tau_{k}(V)=$ $\tau_{k}(W)$, then $O(V, W)=\rho\left(\tau_{k}(V)\right) O\left(\sigma^{k}(V), \sigma^{k}(W)\right)$. We define, for $W$ in $\mathscr{A}^{*}, \mathscr{N}(W)$ as the position of $W$ in the list ordered by < of all words in $\mathscr{A}^{*}$ of length $|W|$. Every word $W$ in $\mathscr{V}^{\#}$ has a least shift, $\mathscr{E}_{-1}(W)$, and a greatest shift, $\mathscr{E}_{+1}(W)$. That is, $\mathscr{E}_{ \pm 1}(W)=\sigma^{k}(W)$ with $k$ taking the value in $\{0, \ldots,|W|-1\}$ such that $O\left(\sigma^{j}(W), \sigma^{k}(W)\right)= \pm 1$ respectively for all $j$ in $\{0, \ldots,|W|-1\} \backslash\{k\}$. The least and greatest shifts are collectively called the extreme shifts. A word $W$ in $\mathscr{V}^{*}$ is maximal if $\mathscr{E}_{ \pm 1}(W)=W$, and minimal if $\mathscr{E}_{-1}(W)=W$. The term $\Omega$-extremal will mean minimal if $\Omega=-1$, and maximal if $\Omega=+1$. Note that $W$ is $\Omega$-extremal if $O\left(\sigma^{k}(W), W\right)=\Omega$ for all $k$ in $\{1, \ldots,|W|-1\}$.

Finally we note that for any true statement about words in $\mathscr{V}^{\#}$ there exists another true statement, its dual, obtained from the former by involution, that is, by switching $L$ and $R, C$ and $K,<$ and $>$, maximal and minimal, and the order of pairs. As an example, the $\chi$-seed $(K, L C)$ would be replaced by the $\chi$-seed $(R K, C)$.

\section{III.1. Proof of Theorem 1.}

III.1.1. The decide-or-depute lemma. Before presenting the principal tool we use in all the proofs, Lemma B, we make several elementary assertions whose proofs are simple. First we give a name to the fact that, in the comparison of a pair of words, what happens after the first discrepancy is of no consequence:

Lemma A1. If $A, B, D$, and $E$ are in $\mathscr{A}^{*} \cup \underline{\mathscr{A}}$, and $\tau_{j}(A) \neq \tau_{j}(B)$ for some $j$ in $\{1, \ldots, \min (|A|,|B|)\}$, then

$$
O(A, B)=O\left(\tau_{j}(A), \tau_{j}(B)\right)=O\left(\tau_{j}(A) D, \tau_{j}(B) E\right) .
$$

Second, we note that when a terminal $Q \in\{C, K\}$ is changed to $Q^{ \pm 1}$, the ordering and parities of the words produced are as given by the following lemma:

Lemma A2. For $U$ in $\mathscr{B}^{*}, Q \in\{C, K\}$,

$$
\begin{gathered}
O\left(U Q^{-\rho(U)} \ldots, U Q\right)=O\left(U Q, U Q^{+\rho(U)} \ldots\right)=1, \\
\rho\left(U Q^{ \pm \rho(U)}\right)= \pm O(M, Q) .
\end{gathered}
$$

And third, we point out the following adjacency property:

Lemma A3. For $U$ in $\mathscr{A}^{*}, Q \in\{C, K\}$, there is no word in $\mathscr{A}^{*}$ of length $|U Q|$ between $U Q^{-1}$ and $U Q$, nor between $U Q$ and $U Q^{+1}$.

We now introduce the decide-or-depute lemma: Lemma $B$. Theorem 1 asserts that certain products of the words $\left\{A P, A P^{+1}, A P^{-1}, B Q, B Q^{+1}, B Q^{-1}\right\}$ (with $A P$ and $B Q$ in $V^{\#}, P$ and $Q$ in $\{C, K\}$ ) are extremal as a consequence of the extremality and mutual bounding properties of the basic words 
$A P$ and $B Q$. Proving it boils down to repeatedly determining the effect, on the order of a pair of words, of replacing a $Q \in\{C, K\}$ in one of them by $Q^{ \pm 1}$. Proving Lemmas D through $\mathrm{G}$ of $\S$ III.2 requires similar determinations. Lemma $\mathrm{B}$, given below, tells us that when the $Q$ is replaced by $Q^{ \pm 1}$ either the order is unchanged, or it is determined in a specified way by the order of the trailing subwords beyond the position of the $Q^{ \pm 1}$.

Lemma B. If $U P$ and $V Q$ are in $\mathscr{V}^{\#}, P$ and $Q$ are in $\{C, K\}$, and $\Omega \equiv$ $O(U P, V Q) \neq 0$, then for any $D$ in $\mathscr{V}^{\#}$,

(i) $O\left(U P^{-\Omega \rho(U)} D, V Q\right)=\Omega$, and either

$$
\tau_{|U P|}(V Q) \neq U P^{+\Omega \rho(U)} \text { and } O\left(U P^{+\Omega \rho(U)} D, V Q\right)=\Omega,
$$

or

and

$$
\tau_{|U P|}(V Q)=U P^{+\Omega \rho(U)}
$$

$$
O\left(U P^{+\Omega \rho(U)} D, V Q\right)=\Omega O(M, P) O\left(D, \sigma^{|U P|}(V Q)\right),
$$

(ii) $O\left(U P, V Q^{+\Omega \rho(V)} D\right)=\Omega$, and either

$$
\tau_{|V Q|}(U P) \neq V Q^{-\Omega \rho(V)} \text { and } O\left(U P, V Q^{-\Omega \rho(V)} D\right)=\Omega,
$$

or

and

$$
\tau_{|V Q|}(U P)=V Q^{-\Omega \rho(V)}
$$

Proof.

$$
O\left(U P, V Q^{-\Omega \rho(V)} D\right)=-\Omega O(M, Q) O\left(\sigma^{|V Q|}(U P), D\right)
$$

(i) Case $|U P|>|V Q| . \quad \tau_{|U P|}(V Q) \neq U P$ because $\left|\tau_{|U P|}(V Q)\right|=|V Q| \neq$ $|U P|$, and $O\left(U P^{ \pm} D, V Q\right)=O(U P, V Q)$ by Lemma $A 1$.

Case $|U P|=|V Q|$.

Subcase $U=V$. Then $P \neq Q$, and $O\left(U P^{ \pm} D, V Q\right)=O(U P, V Q)$ by the observation that $O(C, K)=O\left(C^{ \pm}, K\right)=O\left(C, K^{ \pm}\right)=1$, and Lemma A1.

Subcase $U \neq V . O\left(U P^{ \pm} D, V Q\right)=O(U P, V Q)$ by Lemma A1.

Case $|U P|<|V Q|$.

Subcase $\tau_{|U|}(V) \neq U$. From Lemma $A 1, O\left(U P^{ \pm} D, V Q\right)=O(U P, V Q)$.

Subcase $\tau_{|U|}(V)=U$. First note that $O\left(U P, \tau_{|U P|}(V Q)\right)=O(P, Q)$ by Lemma A1. Therefore, defining $\mathscr{N}(W)$ as the position of $W$ in the ordered list of words in $\mathscr{V}^{\#}$ of length $|W|, \Omega(\mathscr{N}(U P)+\Omega) \leq \Omega \mathscr{N}\left(\tau_{|U P|}(V Q)\right)$.

Now by Lemma A3,

$$
\mathscr{N}\left(U P^{-\rho(U)}\right)+1=\mathscr{N}(U P)=\mathscr{N}\left(U P^{+\rho(U)}\right)-1,
$$

whence

$$
\mathscr{N}\left(U P^{-\Omega \rho(U)}\right)+\Omega=\mathscr{N}(U P)=\mathscr{N}\left(U P^{+\Omega \rho(U)}\right)-\Omega .
$$

Thus

$$
\Omega \mathscr{N}\left(U P^{-\Omega \rho(U)}\right)<\Omega \mathscr{N}\left(\tau_{|U P|}(V Q)\right), \quad \Omega \mathscr{N}\left(U P^{+\Omega \rho(U)}\right) \leq \Omega \mathscr{N}\left(\tau_{|U P|}(V Q)\right) .
$$

Therefore

$$
\begin{aligned}
\Omega & =O\left(U P^{-\Omega \rho(U)}, \tau_{|U P|}(V Q)\right) \\
& =O\left(U P^{-\Omega \rho(U)} D, V Q\right) \text { by Lemma } \mathrm{A} 1
\end{aligned}
$$


If $<$ holds in the last inequality above, we are done, for then $\tau_{|U P|}(V Q) \neq$ $U P^{+\Omega \rho(U)}$, and

$$
\begin{aligned}
\Omega & =O\left(U P^{+\Omega \rho(U)}, \tau_{|U P|}(V Q)\right) \\
& =O\left(U P^{+\Omega \rho(U)} D, V Q\right) \text { by Lemma A1. }
\end{aligned}
$$

Otherwise (equality holds) $\tau_{|U P|}(V Q)=U P^{+\Omega \rho(U)}$, and since

$$
\rho\left(U P^{+\Omega \rho(U)}\right)=\Omega O(M, P) \text { by Lemma A2, }
$$

we have

$$
\begin{aligned}
O\left(U P^{+\Omega \rho(U)} D, V Q\right) & =\rho\left(U P^{+\Omega \rho(U)}\right) O\left(D, \sigma^{|U P|}(V Q)\right) \\
& =\Omega O(M, P) O\left(D, \sigma^{|U P|}(V Q)\right) .
\end{aligned}
$$

(ii) Switch $U P$ and $V Q$ in (i).

III.1.2. Common comparison lemmas. As will be discussed in detail in §III.1.3, the extremality of the emanating words in Theorem 1 is established by comparing each emanating word with each of its shifts. These shifts fall into classes (for example: part of the way through the first A, beginning B, etc.), and each class is treated by the repeated application of the decide-or-depute lemma. To minimize repetition in the proof of Theorem 1, and in those of Lemmas D through G (were we to provide them), we note that several applications of Lemma B often lead to one of a small number of common comparisons, whose outcomes (themselves determined using Lemma B) are given here as Lemmas C. As mentioned earlier, the proofs are quite repetitious and we give only one of them (that of C3a) to serve as an example.

Lemma C1. If $U$ and $V$ are in $\mathscr{B}^{*}, P$ and $Q$ are in $\{C, K\}, D$ is in $\mathscr{V}^{\#}$, and

(i) $O\left(\sigma^{j}(U P), V Q\right)=-O(M, P) \equiv \Omega$ for all $j \in\{0, \ldots,|U|\}$,

(ii) $O\left(\sigma^{k}(V Q), V Q\right)=\Omega$ for all $k \in\{1, \ldots,|V|\}$, then if $\tau_{|V|}(D)=V$,

$$
O\left(\sigma^{i}(U) P^{ \pm} D, V Q\right)=\Omega \text { for all } i \in\{0, \ldots,|U|\} \text {. }
$$

The following instances are perhaps more readable than the lemma itself:

If $A C$ and $B Q$ are in $\mathscr{V}^{\#}, Q$ is in $\{C, K\}, B Q$ is maximal, all shifts of $A C$ are less than $B Q$, and $D$ begins $B$, then $\sigma^{j}(A) C^{ \pm 1} D<B Q$ for all $j \in\{0, \ldots,|A|\}$.

If $A K$ and $B Q$ are in $\mathscr{V}^{\#}, Q$ is in $\{C, K\}, B Q$ is minimal, all shifts of $A K$ are greater than $B Q$, and $D$ begins $B$, then $B Q<\sigma^{j}(A) K^{ \pm 1} D$ for all $j \in\{0, \ldots,|A|\}$.

Lemma C2. If $V Q$ and $D$ are in $\mathscr{V}^{\#}, Q$ is in $\{C, K\}, \tau_{|V|}(D)=V$, and

$$
O\left(\sigma^{i}(V) Q, V Q\right)=-O(M, Q) \equiv \Omega \text { for all } i \in\{1, \ldots,|V|\},
$$

then

$$
O\left(\sigma^{j}(V) Q^{-\Omega \rho(V)} D, V Q\right)=\Omega \text { for all } j \in\{0, \ldots,|V|\} .
$$

Two instances are:

If $A K$ and $D$ are in $\mathscr{V}^{\#}, A K$ is maximal and $D$ begins $A$, then

$$
\sigma^{j}(A) K^{-\rho(A)} D<A K \text { for all } j \in\{0, \ldots,|A|\} .
$$


If $B C$ and $D$ are in $\mathscr{V}^{\#}, B C$ is minimal and $D$ begins $B$, then

$$
B C<\sigma^{j}(B) C^{+\rho(B)} D \text { for all } j \in\{0, \ldots,|B|\} .
$$

Lemma C3. a. If $V Q$ in $\mathscr{V}^{\#}$ is $\Omega$-extremal, where $\Omega=-O(M, Q)$, then

$$
O\left(\sigma^{k}(V)\left[Q^{-\Omega \rho(V)} V\right]^{i} Q, V\left[Q^{-\Omega \rho(V)} V\right]^{j} Q\right)=\Omega
$$

for any $k \in\{1, \ldots,|V|\}, i, j \in\{0,1, \ldots\}$.

b. If $V Q$ is in $\mathscr{V}^{\#}$, and $\Omega=-O(M, Q)$, then

$$
O\left(\left[V Q^{-\Omega \rho(V)}\right]^{i} Q,\left[V Q^{-\Omega \rho(V)}\right]^{j} Q\right)=\Omega,
$$

$i, j \in\{0,1, \ldots\}$ iff $i>j$.

Proof. We arbitrarily choose Lemma C3a for an illustration of the methods of proof used for all the lemmas. Let

$$
\Omega(i, j, k) \equiv O\left(\sigma^{k}(V)\left[Q^{-\Omega \rho(V)} V\right]^{i} Q, V\left[Q^{-\Omega \rho(B)} V\right]^{j} Q\right) .
$$

Then

$$
\begin{aligned}
\Omega(0, j, k) & =O\left(\sigma^{k}(V) Q, V\left[C^{-\rho(V)} V\right]^{j} Q\right) \\
& =O\left(\sigma^{k}(V) Q, \tau_{\left|\sigma^{k}(V) Q\right|}(V)\right) \\
& =O\left(\sigma^{k}(V) Q, V Q\right) \text { by Lemma A1, } \\
& =\Omega \text { by hypothesis. }
\end{aligned}
$$

$$
\begin{aligned}
\Omega(i, 0, k) & =O\left(\sigma^{k}(V)\left[Q^{-\rho(V)} V\right]^{i} Q, V Q\right) \\
& =\Omega \text { by Lemma C } .
\end{aligned}
$$

For $i>0$ and $j>0$, since by Lemma A1

$$
O\left(\sigma^{k}(V) Q, B D\right)=\Omega \text { for any } D \text { in } \mathscr{V}^{\#},
$$

applying Lemma $B$ yields

$$
\Omega(i, j, k)=\Omega \text { if } \rho\left(\sigma^{k}(V)\right)=\rho(V),
$$

and otherwise $\left(\rho\left(\sigma^{k}(V)\right)=-\rho(V)\right)$

$$
\boldsymbol{\Omega}(i, j, k)=\mathbf{\Omega}
$$

unless

$$
\begin{aligned}
\tau_{\left|\sigma^{k}(V) Q\right|}(V) & =\sigma^{k}(V) Q^{+\Omega \rho\left(\sigma^{k}(V)\right)} \\
& =\sigma^{k}(V) Q^{-\Omega \rho(V)}
\end{aligned}
$$

in which case

$$
\begin{aligned}
\Omega(i, j, k) & =\Omega O(M, Q) O\left(V\left[Q^{-\Omega \rho(V)} V\right]^{i-1} Q, \sigma^{k^{\prime}}(V)\left[Q^{-\Omega \rho(B)} V\right]^{j} Q\right), \\
& \text { where } k^{\prime}=\left|\sigma^{k}(V) Q\right|, \\
& =-\Omega^{2}\left(-O\left(\sigma^{k^{\prime}}(V)\left[Q^{-\Omega \rho(B)} V\right]^{j} Q, V\left[Q^{-\Omega(V)} V\right]^{i-1} Q\right)\right) \\
& =\Omega\left(j, i-1, k^{\prime}\right) .
\end{aligned}
$$

By induction on $i, j, \Omega(i, j, k)=\Omega$.

Lemma C4. If $U Q$ and $V Q$ are in $\mathscr{V}^{\#}$, with $Q$ in $\{C, K\}$, and

$$
O\left(\sigma^{i}(V Q), V Q\right)=O\left(\sigma^{j}(U Q), V Q\right)=-O(M, Q) \equiv \Omega
$$


for all $i \in\{1, \ldots,|V|\}, j \in\{0, \ldots,|U|\}$, then for all $n \in\{0,1, \ldots\}$,

$$
O\left(\sigma^{k}(U) Q^{ \pm}\left[V Q^{-\Omega \rho(V)}\right]^{n} V Q,\left[V Q^{-\Omega \rho(V)}\right]^{m} V Q\right)=\Omega
$$

for any $k \in\{0, \ldots,|U|\}, m \in\{0, \ldots, n\}$.

II.1.3. Proof of Theorem 1. We are now in a position to give a proof of the extremality of the emanating words of Theorem 1. The proof is long for three reasons: (i) there are altogether four different types of emanation, and for each type we must compare the emanation with all of its own shifts; (ii) the shifts of each emanation fall into a number of classes each of which must be treated separately; and (iii) each class of shifts must be treated with regard to the various possibilities concerning the relative length of compared subwords. However, the multiplicity of cases is really the only difficulty, and the proof is quite mechanical. Each case is handled by applying Lemma B repeatedly. The set of unresolved cases is systematically reduced, to consist of comparisons of progressively shorter pairs of words. Eventually the only unresolved comparison either is one of those given in the previous section, or involves only the basic words and is directly determined by the defining properties of the seed.

Proof. $\alpha$. Consider $[B]^{n} Q, B$ in $\{L, R\}, Q$ in $\{C, K\}, n$ in $\{0,1, \ldots\}$.

If $n=0,[B]^{n} Q=Q$ is both minimal and maximal.

If $n>0$, for $j$ in $\{1, \ldots, n\}$,

$$
\begin{aligned}
O\left(\sigma^{j}\left([B]^{n} Q\right),[B]^{n} Q\right) & =O\left([B]^{n-j} Q,[B]^{n} Q\right) \\
& =\rho\left([B]^{n-j}\right) O\left(Q,[B]^{j} Q\right)=1 . \\
O(Q, B) & =O(M, B) .
\end{aligned}
$$

Therefore $[B]^{n} Q$ is $O(M, B)$-extremal.

$\psi$. Case $1 \leq k \leq|A|$. We must show that

$$
\begin{aligned}
& O\left(A Q^{ \pm \Omega \rho(A)}\left[B Q^{-\Omega \rho(B)}\right]^{n-1} B Q, \sigma^{k}(A) Q^{ \pm \Omega \rho(A)}\left[B Q^{-\Omega \rho(B)}\right]^{n-1} B Q\right) \\
& \quad=-O(M, Q) \equiv \Omega .
\end{aligned}
$$

By Lemma $A 1$ and hypothesis

$$
O\left(A Q^{ \pm \Omega \rho(A)}\left[B Q^{-\Omega \rho(B)}\right]^{n-1} B Q, \sigma^{k}(A) Q\right)=\Omega .
$$

So by Lemma B(ii),

$$
O\left(A Q^{ \pm \Omega \rho(A)}\left[B Q^{-\Omega \rho(B)}\right]^{n-1} B Q, \sigma^{k}(A) Q^{+\Omega \rho\left(\sigma^{k}(A)\right)}\left[B Q^{-\Omega \rho(B)}\right]^{n-1} B Q\right)=\Omega,
$$

and

$$
O\left(A Q^{ \pm \Omega(A)}\left[B Q^{-\Omega \rho(B)}\right]^{n-1} B Q, \sigma^{k}(A) Q^{-\Omega \rho\left(\sigma^{k}(A)\right)}\left[B Q^{-\Omega \rho(B)}\right]^{n-1} B Q\right)=\Omega
$$

unless

in which case

$$
\tau_{\left|\sigma^{k}(A) Q\right|}\left(A Q^{ \pm \Omega \rho(A)}\left[B Q^{-\Omega \rho(B)}\right]^{n-1} B Q\right)=\sigma^{k}(A) Q^{-\Omega \rho\left(\sigma^{k}(A)\right)}
$$

$$
\begin{aligned}
& O\left(A Q^{ \pm \Omega \rho(A)}\left[B Q^{-\Omega \rho(B)}\right]^{n-1} B Q, \sigma^{k}(A) Q^{-\Omega \rho\left(\sigma^{k}(A)\right)}\left[B Q^{-\Omega \rho(B)}\right]^{n-1} B C\right) \\
&=-\Omega O(M, Q) \\
& \cdot O\left(\sigma^{\left|\sigma^{k}(A) Q\right|}(A) Q^{ \pm \Omega \rho(A)}\left[B Q^{-\Omega \rho(B)}\right]^{n-1} B Q,\left[B Q^{-\Omega \rho(B)}\right]^{n-1} B Q\right), \\
&=(-\Omega)(-\Omega) \Omega \text { by Lemma C4, } \\
&= \Omega .
\end{aligned}
$$


Case $|A|<k$. We must show that, for any $k^{\prime}$ in $\{0, \ldots,|B|\}$, and $m \leq n$,

$$
\begin{aligned}
\Gamma & \equiv O\left(A Q^{ \pm \Omega \rho(A)}\left[B Q^{-\Omega \rho(B)}\right]^{n-1} B Q, \sigma^{k^{\prime}}(B)\left[Q^{-\Omega \rho(B)} B\right]^{m-1} B Q\right) \\
& =-O(M, Q) \equiv \Omega .
\end{aligned}
$$

We have

$$
O\left(A Q, \sigma^{k^{\prime}}(B) Q\right)=-O(M, Q)=\Omega
$$

by hypothesis.

Subcase $|A Q|>\left|\sigma^{k^{\prime}}(B) Q\right|$.

$$
O\left(A Q^{ \pm \Omega(A)}\left[B Q^{-\Omega \rho(B)}\right]^{n-1} B Q, \sigma^{k^{\prime}}(B) Q\right)=\Omega \text { by Lemma A1. }
$$

Thus for $m-1=0, \Gamma=\Omega$. Otherwise $(m-1>0)$, by Lemma $B(i)$ if $\rho\left(\sigma^{k^{\prime}}(B)\right)=-\rho(B), \Gamma=\Omega$ directly.

If $\rho\left(\sigma^{k^{\prime}}(B)\right)=\rho(B)$,

$$
\begin{aligned}
\Gamma & =O\left(A Q^{ \pm \Omega \rho(A)}\left[B Q^{-\Omega \rho(B)}\right]^{n-1} B Q, \sigma^{k^{\prime}}(B)\left[Q^{-\Omega \rho(B)} B\right]^{m-1} Q\right) \\
& =O\left(A Q^{ \pm \Omega \rho(A)}\left[B Q^{-\Omega \rho(B)}\right]^{n-1} B Q, \sigma^{k^{\prime}}(B)\left[Q^{-\Omega\left(\sigma^{k^{\prime}}(B)\right)} B\right]^{m-1} Q\right) \\
& =\Omega
\end{aligned}
$$

unless

$$
\tau_{\left|\sigma^{k^{\prime}}(B) Q\right|}\left(A Q^{ \pm \Omega \rho(A)}\left[B Q^{-\Omega \rho(B)}\right]^{n-1} B Q\right)=\sigma^{k^{\prime}}(B) Q^{-\Omega \rho\left(\sigma^{k^{\prime}}(B)\right)}
$$

in which case

$$
\begin{aligned}
\Gamma & =-\Omega O(M, Q) O\left(\sigma^{\left|\sigma^{k^{\prime}}(B) Q\right|}\left(A Q^{ \pm \Omega \rho(A)}\left[B Q^{-\Omega \rho(B)}\right]^{n-1} B Q\right), B\left[Q^{-\Omega \rho(B)} B\right]^{m-1} Q\right) \\
& =(-\Omega)(-\Omega) \Omega \text { by Lemma C3a, } \\
& =\Omega .
\end{aligned}
$$

Subcase $|A Q|=\left|\sigma^{k^{\prime}}(B) Q\right|$. The hypotheses imply that $O\left(A, \sigma^{k^{\prime}}(B)\right)=\Omega$. So $\Gamma=\Omega$ by Lemma $A 1$.

Subcase $|A Q|<\left|\sigma^{k^{\prime}}(B) Q\right|$. We have

$$
\begin{aligned}
\Omega & =O\left(A Q, \sigma^{k^{\prime}}(B) Q\right) \\
& =O\left(A Q, \sigma^{k^{\prime}}(B)\left[Q^{-\Omega \rho(B)} B\right]^{m-1} Q\right) .
\end{aligned}
$$

So by Lemma $\mathrm{B}(\mathbf{i})$,

$$
O\left(A Q^{-\Omega \rho(A)} \ldots, \sigma^{k^{\prime}}(B)\left[Q^{-\Omega \rho(B)} B\right]^{m-1} Q\right)=\Omega,
$$

and

$$
O\left(A Q^{+\Omega \rho(A)} \ldots, \sigma^{k^{\prime}}(B)\left[Q^{-\Omega \rho(B)} B\right]^{m-1} Q\right)=\Omega
$$

unless $\tau_{|A Q|}\left(\sigma^{k^{\prime}}(B)\right)=A Q^{+\Omega \rho(A)}$, in which case

$$
\begin{aligned}
O\left(A Q^{+\Omega \rho(A)} \ldots, \sigma^{k^{\prime}}(B)\left[Q^{-\Omega \rho(B)} B\right]^{m-1} Q\right) \\
\left.\quad=\Omega O(M, Q) O\left(B Q^{-\Omega \rho(B)}\right]^{n-1} B Q, \sigma^{k^{\prime}+|A Q|}(B)\left[Q^{-\Omega \rho(B)} B\right]^{m-1} Q\right) \\
\quad=\Omega(-\Omega)(-\Omega) \text { by Lemma C3a, } \\
\quad=\Omega .
\end{aligned}
$$

This concludes the proof of part $\psi$.

$\chi$. Let $(A K, B C)$ be a $\chi$-seed. 
(i) We show that $\beta_{(+1, \pm 1)}(A K, B C)$ are maximal. That is, we show that $O\left(\sigma^{k}\left(A K^{ \pm \rho(A)} B C\right), A K^{ \pm \rho(A)} B C\right)=1$, for any $k$ in $\left\{1, \ldots,\left|A K^{ \pm} B\right|\right\}$.

Case $1 \leq k \leq|A|$ :

$$
O\left(\sigma^{k}\left(A K^{ \pm \rho(A)} B C\right), A K^{ \pm \rho(A)} B C\right)=O\left(\sigma^{k}(A) K^{ \pm \rho(A)} B C, A K^{ \pm \rho(A)} B C\right) .
$$

Since $O\left(\sigma^{k}(A) K, A K^{ \pm \rho(A)} B C\right)=1$ (by hypothesis because $\left|\sigma^{k}(A)\right|<|A|$ ), by Lemma $B(i)$ we have

$$
O\left(\sigma^{k}(A) K^{-\rho\left(\sigma^{k}(A)\right)} B C, A K^{ \pm \rho(A)} B C\right)=1,
$$

and

$$
O\left(\sigma^{k}(A) K^{+\rho\left(\sigma^{k}(A)\right)} B C, A K^{ \pm \rho(A)} B C\right)=1
$$

unless

$$
\tau_{\left|\sigma^{k}(A) K\right|}\left(A K^{ \pm \rho(A)} B C\right)=\sigma^{k}(A) K^{+\rho\left(\sigma^{k}(A)\right)}
$$

in which case

$$
\begin{aligned}
O\left(\sigma^{k}\right. & \left.(A) K^{+\rho\left(\sigma^{k}(A)\right)} B C, A K^{ \pm \rho(A)} B C\right) \\
& =+1 . O(M, K) O\left(B C, \sigma^{\left|\sigma^{k}(A) K\right|}\left(A K^{ \pm \rho(A)} B C\right)\right) \\
& =O\left(B C, \sigma^{\left|\sigma^{k}(A) K\right|}(A) K^{ \pm \rho(A)} B C\right) \\
& =1, \text { by Lemma C1. }
\end{aligned}
$$

Case $k=|A K| . O\left(B C, A K^{ \pm \rho(A)} B C\right)=1$, by Lemma $\mathrm{C} 1$.

Case $|A K|<k$. We must show that $O\left(\sigma^{k^{\prime}}(B C), A K^{ \pm} B C\right)=1$ for $k^{\prime}$ in $\{0, \ldots,|B|\}$.

Since $O\left(\sigma^{k^{\prime}}(B C), A K\right)=1$, then by Lemma $\mathrm{B}(\mathrm{ii})$

$$
O\left(\sigma^{k^{\prime}}(B C), A K^{+\rho(A)} B C\right)=1,
$$

and

$$
O\left(\sigma^{k^{\prime}}(B C), A K^{-\rho(A)} B C\right)=1,
$$

unless

$$
\tau_{|A K|}\left(\sigma^{k^{\prime}}(B C)\right)=A K^{-\rho(A)}
$$

in which case

$$
\begin{aligned}
O\left(\sigma^{k^{\prime}}(B C), A K^{-\rho(A)} B C\right) & =-1 \cdot O(M, K) O\left(\sigma^{|A K|}\left(\sigma^{k^{\prime}}(B C)\right), B C\right) \\
& =-(-1)=1 .
\end{aligned}
$$

That $\beta_{(-1, \pm 1)}(A K, B C)$ are minimal is the dual of the statement just proved.

(ii) We show that for all $n$ in $\{1,2, \ldots\}, \chi_{(-1, \pm n)}(A K, B C)$ are minimal. The following discussion applies to any fixed $n$. Setting $V C \equiv A K^{ \pm \rho(A)} B C$, we must show that

$$
\Delta_{j} \equiv O\left(B C^{-\rho(B)}\left[V C^{-\rho(V)}\right]^{n-1} V C, \sigma^{j}\left(B C^{-\rho(B)}\left[V C^{-\rho(V)}\right]^{n-1} V C\right)\right)=1
$$

for all $j$ in $\left\{1, \ldots,\left|B C^{-\rho(B)}\left[V C^{-\rho(V)}\right]^{n-1} V\right|\right\}$.

Case $1 \leq j \leq|B|$. Then

$$
\Delta_{j}=O\left(B C^{-\rho(B)}\left[V C^{-\rho(V)}\right]^{n-1} V C, \sigma^{j}(B) C^{-\rho(B)}\left[V C^{-\rho(V)}\right]^{n-1} V C\right) .
$$


We have $O\left(B C^{-\rho(B)}, \sigma^{j}(B) C\right)=1$, by hypothesis and Lemma $\mathrm{Al}$, so by Lemma B(ii) $\Delta_{j}=1$ directly or $\left(\rho\left(\sigma^{j}(B)\right)=\rho(B)\right.$ and)

$$
\begin{aligned}
\Delta_{j} & =-1 \cdot O(M, C) \cdot O\left(\sigma^{\left|\sigma^{j}(B) C\right|}(B) C^{-\rho(B)}\left[V C^{-\rho(V)}\right]^{n-1} V C,\left[V C^{-\rho(V)}\right]^{n-1} V C\right), \\
& =-1 \cdot O(M, C) \cdot 1, \quad \text { by Lemma } C 4 \text { and Theorem } 1 \chi(\mathrm{i}), \\
& =1 .
\end{aligned}
$$

Case $j>|B|$. Then either $\Delta_{j}=O\left(B C^{-\rho(B)}\left[V C^{-\rho(V)}\right]^{n-1} V C, \sigma^{j^{\prime}}(V) C\right)=1$ by hypothesis, or

$$
\Delta_{j}=\Delta_{j^{\prime}}^{\prime} \equiv O\left(B C^{-\rho(B)}\left[V C^{-\rho(V)}\right]^{n-1} V C, \sigma^{j^{\prime}}(V) C^{-\rho(V)}\left[V C^{-\rho(V)}\right]^{m-1} V C\right)
$$

for some $j^{\prime}$ in $\{0, \ldots,|V|\}$ and $m$ in $\{1, \ldots, n-1\}$.

Subcase $j^{\prime}=|A K|$.

$$
\Delta=O\left(B C^{-\rho(B)}\left[V C^{-\rho(V)}\right]^{n-1} V C, B C^{-\rho(V)}\left[V C^{-\rho(V)}\right]^{m-1} V C\right) .
$$

Subsubcase $\rho(B)=-\rho(V)$.

$$
\Delta_{j^{\prime}}^{\prime}=1 \quad \text { since } O\left(B C^{-\rho(B)}, B C^{+\rho(B)}\right)=1 .
$$

Subsubcase $\rho(B)=\rho(V)$.

$$
\begin{aligned}
\Delta_{j^{\prime}}^{\prime} & =O\left(B C^{-\rho(B)}\right) O\left(\left[V C^{-\rho(V)}\right]^{n-1} V C,\left[V C^{-\rho(V)}\right]^{m-1} V C\right), \\
& =O\left(B C^{-\rho(B)}\right) \cdot 1, \quad \text { by Lemma C3b, } \\
& =1 .
\end{aligned}
$$

Subcase $j^{\prime}>|A K|$. Then

$$
\Delta_{j^{\prime}}^{\prime}=\Delta_{j^{\prime \prime}}^{\prime \prime} \equiv O\left(B C^{-\rho(B)}\left[V C^{-\rho(V)}\right]^{n-1} V C, \sigma^{j^{\prime \prime}}(B) C^{-\rho(V)}\left[V C^{-\rho(V)}\right]^{m-1} V C\right) \text {, }
$$

for some $j^{\prime \prime}$ in $\{1, \ldots,|B|\}$. We have by hypothesis and Lemma A1

$$
O\left(B C^{-\rho(B)}\left[V C^{-\rho(V)}\right]^{n-1} V C, \sigma^{j^{\prime \prime}}(B) C\right)=1 .
$$

Subcase $\rho(B)=-\rho(V) . \Delta_{j^{\prime \prime}}^{\prime \prime}=1$ by Lemma B(ii).

Subcase $\rho(B)=\rho(V)$. By Lemma $B(i i) \Delta_{j^{\prime \prime}}^{\prime \prime}=1$ directly, or

$$
\begin{aligned}
\Delta_{j^{\prime \prime}}^{\prime \prime} & =O\left(\sigma^{\left|\sigma^{j \prime \prime}(B) C\right|}(B) C^{-\rho(B)}\left[V C^{-\rho(V)}\right]^{n-1} V C,\left[V C^{-\rho(V)}\right]^{m-1} V C\right) \\
& =1 \text { by Lemma C4. }
\end{aligned}
$$

Case $0 \leq j^{\prime} \leq|A|$. Then

$$
\Delta_{j^{\prime}}^{\prime}=O\left(B C^{-\rho(B)}\left[V C^{-\rho(V)}\right]^{n-1} V C, \sigma^{j^{\prime}}(A) K^{- \pm \rho(A)} B C^{ \pm \rho(B)}\left[V C^{-\rho(V)}\right]^{m-1} V C\right) .
$$

Subcase $\left|\sigma^{j^{\prime}}(A)\right|<|B|$. We have by hypothesis and Lemma $\mathrm{Al}$

$$
O\left(B C^{-\rho(B)}\left[V C^{-\rho(V)}\right]^{n-1} V C, \sigma^{j^{\prime}}(A) K\right)=1 .
$$

Therefore by Lemma $B$, $O\left(B C^{-\rho(B)}\left[V C^{-\rho(V)}\right]^{n-1} V C, \sigma^{j^{\prime}}(A) K^{+\rho\left(\sigma^{j^{\prime}}(A)\right)} B C^{ \pm \rho(B)}\left[V C^{-\rho(V)}\right]^{m-1} V C\right)=1$, and

$$
O\left(B C^{-\rho(B)}\left[V C^{-\rho(V)}\right]^{n-1} V C, \sigma^{j^{\prime}}(A) K^{-\rho\left(\sigma^{\prime}(A)\right)} B C^{ \pm \rho(B)}\left[V C^{-\rho(V)}\right]^{m-1} V C\right)=1
$$


directly or

$$
\begin{aligned}
= & -1 \cdot O(M, K) \cdot O\left(\sigma^{j^{\prime \prime \prime}}(B) C^{-\rho(B)}\left[V C^{-\rho(V)}\right]^{n-1} V C,\right. \\
& \left.B C^{ \pm \rho(B)}\left[V C^{-\rho(V)}\right]^{m-1} V C\right) \\
= & O\left(B C^{ \pm \rho(B)}\left[V C^{-\rho(V)}\right]^{m-1} V C, \sigma^{j^{\prime \prime \prime}}(B) C^{-\rho(B)}\left[V C^{-\rho(V)}\right]^{n-1} V C\right),
\end{aligned}
$$

for some $j^{\prime \prime \prime}$ in $\left\{1, \ldots,\left|\sigma^{j^{\prime}}(A) K\right| \leq|B|\right\}$.

Now we have

$$
O\left(B C^{ \pm \rho(B)}\left[V C^{-\rho(V)}\right]^{m-1} V C, \sigma^{j^{\prime \prime \prime}}(B) C\right)=1
$$

by hypothesis and Lemma A1, so by Lemma $B$

$$
O\left(B C^{ \pm \rho(B)}\left[V C^{-\rho(V)}\right]^{m-1} V C, \sigma^{j^{\prime \prime \prime}}(B) C^{+\rho\left(\sigma^{j \prime \prime \prime}(B)\right)}\left[V C^{-\rho(V)}\right]^{n-1} V C\right)=1,
$$

and

$$
O\left(B C^{ \pm \rho(B)}\left[V C^{-\rho(V)}\right]^{m-1} V C, \sigma^{j^{\prime \prime \prime}}(B) C^{-\rho\left(\sigma^{j \prime \prime \prime}(B)\right)}\left[V C^{-\rho(V)}\right]^{n-1} V C\right)=1,
$$

directly or

$$
\begin{aligned}
& =-1 \cdot O(M, C) \cdot O\left(\sigma^{j^{\prime \prime \prime}}(B) C^{ \pm(B)}\left[V C^{-\rho(V)}\right]^{m-1} V C,\left[V C^{-\rho(V)}\right]^{n-1} V C\right), \\
& =O\left(\sigma^{j^{\prime \prime \prime}}(B) C^{ \pm \rho(B)}\left[V C^{-\rho(V)}\right]^{m-1} V C,\left[V C^{-\rho(V)}\right]^{n-1} V C\right) .
\end{aligned}
$$

Now we have $O\left(\sigma^{j^{\prime \prime \prime}}(B) C,\left[V C^{-\rho(V)}\right]^{n-1} V C\right)=1$ by hypothesis and Lemma A1, so

$$
O\left(\sigma^{j^{\prime \prime \prime}}(B) C^{-\rho\left(\sigma^{j \prime \prime}(B)\right)}\left[V C^{-\rho(V)}\right]^{m-1} V C,\left[V C^{-\rho(V)}\right]^{n-1} V C\right)=1,
$$

and

$$
O\left(\sigma^{j^{\prime \prime \prime}}(B) C^{+\rho\left(\sigma^{\prime \prime \prime \prime}(B)\right)}\left[V C^{-\rho(V)}\right]^{m-1} V C,\left[V C^{-\rho(V)}\right]^{n-1} V C\right)=1
$$

directly or

$$
=+1 \cdot O(M, C) \cdot O\left(\left[V C^{-\rho(V)}\right]^{m-1} V C, \sigma^{j^{\prime \prime \prime \prime}}(V) C^{-\rho(V)}\left[V C^{-\rho(V)}\right]^{n^{\prime}-1} V C\right) \text {, }
$$

for some $n^{\prime} \geq m$, and where $j^{\prime \prime \prime \prime}=\left|\sigma^{j^{\prime \prime \prime}}(B) C\right|$,

$$
\begin{aligned}
& =-1 \cdot-1 \text { by Lemma C3a, } \\
& =1 .
\end{aligned}
$$

Subcase $\left|\sigma^{j^{\prime}}(A)\right|=|B|$. We have $O\left(B C, \sigma^{j^{\prime}}(A) K\right)=1$ by hypothesis, and so

$$
O\left(B C^{-\rho(B)}\left[V C^{-\rho(V)}\right]^{n-1} V C, \sigma^{j^{\prime}}(A) K\right)=1 .
$$

Then by Lemma $B(i i)$

$$
\begin{aligned}
& O\left(B C^{-\rho(B)}\left[V C^{-\rho(V)}\right]^{n-1} V C, \sigma^{j^{\prime}}(A) K^{+\rho\left(\sigma^{j^{\prime}}(A)\right)} B C^{ \pm \rho(B)}\left[V C^{-\rho(V)}\right]^{m-1} V C\right) \\
& \quad=1
\end{aligned}
$$

and

$$
\begin{aligned}
& O\left(B C^{-\rho(B)}\left[V C^{-\rho(V)}\right]^{n-1} V C, \sigma^{j^{\prime}}(A) K^{-\rho\left(\sigma^{j^{\prime \prime \prime}}(B)\right)} B C^{ \pm \rho(B)}\left[V C^{-\rho(V)}\right]^{m-1} V C\right) \\
& \quad=1
\end{aligned}
$$


directly or

$$
\begin{aligned}
& =-O(M, K) O\left(\left[V C^{-\rho(V)}\right]^{n-1} V C, B C^{ \pm \rho(B)}\left[V C^{-\rho(V)}\right]^{m-1} V C\right) \\
& =O\left(B C^{ \pm \rho(B)}\left[V C^{-\rho(V)}\right]^{m-1} V C,\left[V C^{-\rho(V)}\right]^{n-1} V C\right) .
\end{aligned}
$$

Now $O\left(B C,\left[V C^{-\rho(V)}\right]^{n-1} V C\right)=1$ by hypothesis and Lemma $A 1$, so

$$
\begin{gathered}
O\left(B C^{-\rho(B)}\left[V C^{-\rho(V)}\right]^{m-1} V C,\left[V C^{-\rho(V)}\right]^{n-1} V C\right)=1, \quad \text { and } \\
O\left(B C^{+\rho(B)}\left[V C^{-\rho(V)}\right]^{m-1} V C,\left[V C^{-\rho(V)}\right]^{n-1} V C\right)=1
\end{gathered}
$$

unless $B C^{+\rho(B)}=\tau^{|B C|}(V)$, in which case

$$
\begin{aligned}
& O\left(B C^{+\rho(B)}\left[V C^{-\rho(V)}\right]^{m-1} V C,\left[V C^{-\rho(V)}\right]^{n-1} V C\right) \\
& =O(M, C) O\left(\left[V C^{-\rho(V)}\right]^{m-1} V C, \sigma^{|B C|}(V) C^{-\rho(V)}\left[V C^{-\rho(V)}\right]^{n-2} V C\right) \\
& \text { if } n>1 \text {, } \\
& =-1 \cdot-1=1 \text { by Lemma C3a, }
\end{aligned}
$$

or, if $n=1$,

$$
=O(M, C) O\left(\left[V C^{-\rho(V)}\right]^{m-1} V C, \sigma^{|B C|}(V) C\right)=1 \text { by hypothesis. }
$$

Subcase $\left|\sigma^{j^{\prime}}(A)\right|>|B|$. We have by hypothesis and Lemma A1

$$
O\left(B C, \sigma^{j^{\prime}}(A) K^{- \pm \rho(A)} B C^{ \pm \rho(B)}\left[V C^{-\rho(V)}\right]^{m-1} V C\right),
$$

so by Lemma $B$

$$
\begin{aligned}
\Delta & =O\left(B C^{-\rho(B)}\left[V C^{-\rho(V)}\right]^{n-1} V C, \sigma^{j^{\prime}}(A) K^{- \pm \rho(A)} B C^{ \pm \rho(B)}\left[V C^{-\rho(V)}\right]^{m-1} V C\right) \\
& =1 .
\end{aligned}
$$

That $\chi_{(+1, \pm n)}(A K, B C)$ are maximal is the dual of the statement just proven.

\section{III.2. Proof of Theorem 2.}

III.2.1. Extreme-shift lemmas. The proof of Theorem 2, to be given shortly, is constructive. It uses a deterministic algorithm (the Retraction Algorithm; see III.2.4) to effect a decomposition of an extremal word in $\mathscr{V}^{\#}$ that reveals its seed of origin. The "retraction" to the seed entails the repeated truncation of the word-deletion of the extreme shift-and it relies both on the extremality of the word remaining at each stage of the process, and on knowledge of the extreme shift of the remaining word. The first lemma in this section identifies the extreme shifts of the extremal words that are specified as emanations in Theorem 1. Those that follow assure the extremality of the words that remain after each truncation, and enable us to identify the extreme shifts of those words.

Lemma D. $\alpha$. For each $n$ in $\{1,2, \ldots\}, \mathscr{E}_{+1}\left([L]^{n-1} C\right)=C, \mathscr{E}_{-1}\left([R]^{n-1} K\right)$ $=K$.

$\psi$. If $(U Q, V Q)$ is a $\psi$-seed, then with $\Omega=-O(M, Q)$, for each $n$ in $\{1,2, \ldots\}, \mathscr{E}_{\Omega}\left(\psi_{ \pm n}(U Q, V Q)\right)=V Q$.

$\chi$. If $(A K, B C)$ is a $\chi$-seed, then for each $n$ in $\{1,2, \ldots\}$,

the greatest shift of $\beta_{(-1, \pm 1)}(A K, B C)$ is $A K$,

the greatest shift of $\chi_{(-1, \pm n)}(A K, B C)$ is $A K^{- \pm \rho(A)} B C$,

the least shift of $\beta_{(+1, \pm 1)}(A K, B C)$ is $B C$, and 
the least shift of $\chi_{(+1, \pm n)}(A K, B C)$ is $B C^{ \pm \rho(B)} A K$.

Lemma E1. a. If $U X V C$, with $X \in \mathscr{B}$, is in $\mathscr{V}^{\#}$ and is minimal, and $V C$ is its greatest shift, then $U C$ is minimal.

b. If $U X V C$, with $X \in \mathscr{B}$, is in $\mathscr{V}^{\#}$ and maximal, and $V C$ is its least shift, then $U K$ is maximal.

Lemma E2. If $U V X V C$, with $X \in \mathscr{B}$, is in $\mathscr{V}^{\#}$ and minimal with greatest shift $V C$, then $U V C$ is minimal with greatest shift $V C$.

Lemma E3. If $U Y V X U C$, with $X, Y \in \mathscr{B}$, is in $\mathscr{V}^{\#}$ and minimal with greatest shift $V X U C$, then $U C$ is the least shift of $V X U C$.

III.2.2. Uniqueness lemma. The Retraction Algorithm (III.2.4) ignores the letter that precedes the extreme shift at each stage of the decomposition, except the last. The following lemma is needed to show that knowledge of those letters is nevertheless not lost. The reason is that the extremal words specified in Theorem 1 are unique in the following senses.

Lemma F. $\alpha .[L]^{n} C$ is the only word in $\mathscr{V}^{\#}$ of length $\left|[L]^{n} C\right|$ with greatest shift $C, n \in\{0,1, \ldots\}$, and $[R]^{n} K$ is the only word in $\mathscr{V}^{\#}$ of length $\left|[R]^{n} K\right|$ with least shift $K$.

$\psi$. If $(A Q, B Q)$ is a $\psi$-seed, the words $\Psi_{n}^{ \pm}(A Q, B Q)$ defined in Theorem 1 are the only words in $\mathscr{V}^{\#}$ of the form $A X_{0} B X_{1} B X_{1} \cdots B X_{n} B Q$, with $X_{i} \in \mathscr{B}$ for which

$$
\mathscr{E}_{-O(M, Q)}\left(A X_{0} B X_{1} B X_{1} \cdots B X_{n} B Q\right)=B Q .
$$

$\chi$. If $(A K, B C)$ is a $\chi$-seed, then

$$
\chi_{(-1, \pm n)}(A K, B C), \quad n \in\{1,2, \ldots\},
$$

are the only minimal words in $\mathscr{V}^{\#}$ of the form

$$
W_{\mathbf{x}}^{ \pm}=B X_{0} A K^{ \pm 1} B X_{1} A K^{ \pm 1} B X_{2} \cdots A K^{ \pm 1} B X_{n-1} A K^{ \pm 1} B C
$$

for which $\mathscr{E}_{+1}\left(W_{\mathbf{x}}^{ \pm}\right)=A K^{+\rho(A)} B C$, and

$$
\chi_{(+1, \pm n)}(A K, B C), \quad n \in\{1,2, \ldots\},
$$

are the only maximal words in $\mathscr{V}^{\#}$ of the form

$$
W_{\mathbf{x}}^{ \pm}=A X_{0} B C^{ \pm 1} A X_{1} B C^{ \pm 1} A X_{2} \cdots B C^{ \pm 1} A X_{n-1} B C^{ \pm 1} A K
$$

for which $\mathscr{E}_{-1}\left(W_{\mathbf{x}}^{ \pm}\right)=B C^{-\rho(B)} A K$.

III.2.3. Seed existence lemmas. The Retraction Algorithm eventually generates a pair of words in $\mathscr{V}^{\#},(U P, V Q)$, with $P, Q \in\{C, K\}$, which is asserted to be the seed from which the original word emanates. The following lemmas are required to ensure that the pair generated does in fact have the defining properties of a seed of the appropriate type.

Lemma G1. a. If $A X B C$ in $\mathscr{V}^{\#, ~ w i t h ~} X \in \mathscr{B}$, has least shift $B C$, then $O\left(B C, \sigma^{j}(A K)\right)=1$ for all $j \in\{0, \ldots,|A|\}$.

b. If $A X B C$ in $\mathscr{V}^{\#}$, with $X \in \mathscr{B}$, is maximal with lecst shift $B C$, then $O\left(\sigma^{j}(B C), A K\right)=1$ for all $j \in\{0, \ldots,|B|\}$.

Lemma G2. a. If $A X B C$ in $\mathscr{V}^{\#}$, with $X \in \mathscr{B}$, is minimal with greatest shift $B C$, and $A$ is not a shift of $B$, then $O\left(A C, \sigma^{j}(B C)\right)=1$ for all $j \in$ $\{0, \ldots,|B|\}$. 
b. If $A X B C$ in $\mathscr{V}^{\#}$, with $X \in \mathscr{B}$, is minimal with greatest shift $B C$, and $B$ is not a shift of $A$, then $O\left(\sigma^{j}(A C), B C\right)=1$ for all $j \in\{0, \ldots,|A|\}$.

Lemma H1. If $A X B C$ in $\mathscr{V}^{\#}$, with $X \in \mathscr{A}^{\prime}$, is maximal with least shift $B C$, then $(A K, B C)$ is a $\chi$-seed.

Proof. $B C$ is minimal by hypothesis.

$A K$ is maximal by Lemma $\mathrm{E} 1 \mathrm{~b}$.

$O\left(B C, \sigma^{j}(A K)\right)=1$ for all $j \in\{0, \ldots,|A|\}$ by Lemma Gla.

$O\left(\sigma^{j}(B C), A K\right)=1$ for all $j \in\{0, \ldots,|B|\}$ by Lemma G1b.

Lemma H2. If $A X B C$ in $\mathscr{V}^{\#}$, with $X \in \mathscr{B}$, is minimal with greatest shift $B C$, and neither $A$ nor $B$ is a shift of the other, then $(B C, A C)$ is a $\psi$-seed.

Proof. $B C$ is maximal by hypothesis.

$A C$ is minimal by Lemma E1a.

$O\left(\sigma^{j}(A C), B C\right)=1$ for all $j \in\{0, \ldots,|A|\}$ by Lemma G2b.

$O\left(A C, \sigma^{j}(B C)\right)=1$ for all $j \in\{0, \ldots,|B|\}$ by Lemma G2a.

II.2.4. Proof of Theorem 2. We are finally in a position to present a proof of our result that every word in $\mathscr{V}^{\#}$ emanates from a unique seed. Given a word in $\mathscr{V}^{\#}$, the algorithm given below determines a seed from which the word emanates, as well as the complete specification of the emanation. The letters $X_{1}, X_{2}, \ldots, X_{n}$ of Lemma $\mathrm{F}$ are ignored by the algorithm, but by that lemma, those letters are determined by $X_{0}$ which is not ignored. Existence of a seed of origin is thus constructively demonstrated. Using Lemma D, it is easily checked that all the emanations specified in Theorem 1 are correctly decomposed by the algorithm. That is, the $A$ and $B$ words are recovered, the type of seed is identified, and the type and indices of the emanation are determined correctly. Uniqueness therefore follows from the fact that if a word emanates from the seed $(A P, B Q)$ and from the seed $\left(A^{\prime} P^{\prime}, B^{\prime} Q^{\prime}\right)$, then the algorithm recovers $(A P, B Q)$ and it recovers $\left(A^{\prime} P^{\prime}, B^{\prime} Q^{\prime}\right)$. Since the algorithm is deterministic, $(A P, B Q)=\left(A^{\prime} P^{\prime}, B^{\prime} Q^{\prime}\right)$.

Retraction Algorithm. The seed from which a word in $\mathscr{V}^{\#}$ emanates is determined by applying the following algorithm.

Case: The word is of the form $W C$.

Case: $W C$ is maximal.

Case: $|W|=0 . W C=C$ is also minimal, and $W C=\alpha_{-1}$.

Case: $|W| \neq 0$. Define $U, V$ in $\mathscr{B}^{*}, X$ in $\mathscr{B}$, by $W C=$ $U X V C$, where $V C$ is the least shift of $W C$. Then by Lemma $\mathrm{H} 1,(U K, V C)$ is a $\chi$-seed, and

$$
W C=\beta_{(+1, \rho(U X))}(U K, V C) \text {. }
$$

Case: $W C$ is minimal.

I. If the greatest shift of $W C$ is $C$, then $W C=\alpha_{-|W C|}$, by Lemma $F \alpha$. Otherwise set $T C=W C$, and $V C=E_{+1}(W C)$ which is maximal.

II. If $V C$ is not a shift of $T C$, go to step III. Otherwise, define $U X, X$ in $\mathscr{B}$, by $T C=U X V C$. By Lemma E1, $U C$ is minimal. By Lemma E2, if $V C$ is a shift of $U C$, it is its greatest shift. Redefine $T$ by $T C=U C$ and repeat step II. 
III. Let $n$ be the number of times the body of step II was performed. If $T C$ is a shift of $V C$, it is its least shift by Lemma E3. Therefore defining $S X$, with $X$ in $\mathscr{B}$, by $V C=S X T C$, $(S K, T C)$ is a $\chi$-seed by Lemma $\mathrm{H} 1$, and

$$
W C=\chi_{(-1,-\rho(S X) n)}(S K, T C) \text {. }
$$

Otherwise, neither $T C$ nor $V C$ is a shift of the other, whence by Lemma $\mathrm{H} 2,(V C, T C)$ is a $\psi$-seed, and

$$
W C=\Psi_{-\rho(T X) n}(V C, T C) .
$$

(Note: $T C$ cannot become empty by removal of $V C$ because $T C \neq V C$ since one is maximal and the other is minimal, and $V C$ is not the one word, $C$, that is both. That possibility was disposed of in step I.)

Case: The word is of the form $W K$. The seed is determined by the dual under involution of the algorithm above.

Examples. (i) We apply the algorithm to the word $L L M M M C$, which is minimal.

I. Set $T C=L L M M M C$, and $V C=M C$ the greatest shift.

II. $V C$ is a shift of $T C$, so set $U X=L L M M$, and redefine $T C=U C=$ $L L M C$.

$V C$ is a shift of $T C$, so set $U X=L L$, and redefine $T C=U C=L C$.

$V C$ is not a shift of $T C$, so proceed to step III. +1 .

III. Now $V C=M C, T C=L C, X=L, n=2$, and $\rho(T X)=\rho(L L)=$

$T C$ is not a shift of $V C$; therefore $L L M M M C=\Psi_{-2}(M C, L C)$.

(ii) We apply the algorithm to the word $L M C$, which is minimal.

I. Set $T C=L M C$, and $V C=M C$ the greatest shift.

II. $V C$ is a shift of $T C$, so set $U X=L$, and redefine $T C=U C=C$.

$V C=M C$ is not a shift of $T C=C$, so proceed to step III.

III. Now $V C=M C, T C=C, X=L, n=1$, and $\rho(T X)=\rho(L)=+1$. $T C$ is a shift of $V C$, so set $S X$ by $M C=S X T C$ (that is, $S=1$ and $X=M)$ and so $L M C=\chi_{(-1,-1)}(K, C)$.

\section{A NEW PARTIAL ORDER ON FINITE KNEADING SEQUENCES}

Theorems 1 and 2 yield directly a new partial order on finite kneading sequences that we formalize as follows:

- say $K_{1}$ is a strict parent of $K_{2}$ if $K_{2}$ emanates from a seed of the form $\left(K_{0}, K_{1}\right)$ or $\left(K_{1}, K_{0}\right)$, (by Theorem 2 , each finite kneading sequence has exactly two strict parents);

- say $K_{1}$ is a parent of $K_{2}$, in symbols $K_{1} \ll K_{2}$, if $K_{1}$ is a strict parent of $K_{2}$, or $K_{1}=K_{2}$;

- say $K_{1}$ is an ancestor of $K_{n}$, in symbols $K_{1} \ll \ll K_{n}$, if there exists a sequence $\left(K_{1}, K_{2}, \ldots, K_{n}\right)$, with $n \geq 2$, such that $K_{i} \ll K_{i+1}$ for $1 \leq i \leq$ $n-1$.

Defining the topological entropy $h(K)$ of a kneading sequence $K$ as the infimum of topological entropies of maps $f$ such that $K$ is a kneading sequence of $f$, we have: 
Theorem 3. $K_{1} \ll \ll K_{2} \Rightarrow h\left(K_{1}\right) \leq h\left(K_{2}\right)$.

The two-parameter family of stunted saw-tooth maps is defined in the next section. Since the kneading data of a map determines its topological entropy [MT], and since any kneading data for $\{+-+\}$-bimodal maps are realized by some stunted saw-tooth map, we can also define the topological entropy $h(K)$ of a kneading sequence $K$ as the infimum of topological entropies of stunted saw-tooth maps $T_{a, b}^{+}$such that $K$ is a kneading sequence of $T_{a, b}^{+}$. The proof of Theorem 3 will be given in the next section, using this alternate but equivalent definition of $h(K)$.

\section{STUNTED SAW-TOOTH MAPS}

We set define the full $\{+-+\}$-bimodal stunted saw-tooth map $S^{+}$as:

$$
S^{+}= \begin{cases}5 x & \text { if } 0 \leq x \leq \frac{1}{5} \\ 5\left(\frac{3}{5}-x\right) & \text { if } \frac{2}{5} \leq x \leq \frac{3}{5} \\ 5\left(x-\frac{4}{5}\right) & \text { if } \frac{4}{5} \leq x \leq 1 \\ 1 & \text { if } \frac{1}{5} \leq x \leq \frac{2}{5} \\ 0 & \text { if } \frac{3}{5} \leq x \leq \frac{4}{5}\end{cases}
$$

(The symbol $S^{-}$will be reserved for the analogous $\{+-+\}$-bimodal map, treated elsewhere [R].) The graph of $S^{+}$is a succession of a hill and a valley. It is easy to check that the set of itineraries for this map is the set of all possible itineraries for $\{+-+\}$-bimodal maps. We can now define a two-parameter family of $\{+-+\}$-bimodal stunted saw-tooth maps $T_{a, b}^{+}$by setting

$$
T_{a, b}^{+}(x)= \begin{cases}\min \left(S^{+}(x), a\right) & \text { on the hill, } \\ \max \left(S^{+}(x), 1-b\right) & \text { in the valley. }\end{cases}
$$

For the map $T_{a, b}^{+}$to be an endomorphism of the unit interval imposes the constraints

$$
0 \leq a \leq 1, \quad 0 \leq b \leq 1, \quad a+b \geq 1 .
$$

The set of parameter values $(a, b)$ satisfying $(*)$ is denoted by $\mathbf{P}^{+}$. Figure 1.0 shows that the graph of $S^{+}$and the parameter space $\mathrm{P}^{+}$. All kneading
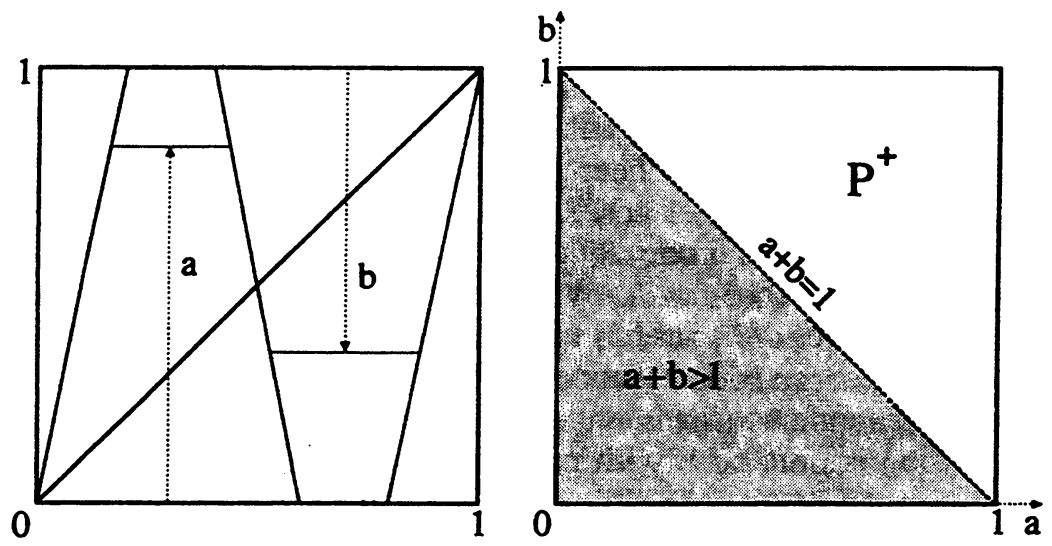

FIGURE 1.0. Graph and parameter space of the stunted saw-tooth family $T_{a, b}^{+}$ 
sequences of the truncated maps $T_{a, b}^{+}$can be understood as the itineraries under $S^{+}$of those points which are closer to one of the turning points than any other point in their orbit. This makes the study of these maps quite simple: the arguments presented in [GT] and [BMT] for stunted maps in the unimodal case extend easily; in particular, any $\{+-+\}$ kneading data occur for some pair $(a, b)$. More on general stunted families will be reported in [DGMT]; here we use these maps for the sake of illustration and to provide a geometric picture to display the results of Theorems 2 and 3.

In $\mathbf{P}^{+}$, following [MaT1-MaT3], [M], and [RS1, RS2] we define subsets corresponding to finite kneading sequences, which can be described as follows (proofs are straightforward and left to the reader):

- Any finite maximal kneading sequence terminating $K$ is realised on a single segment of straight line parallel to the $a$-axis, with one external end point on the line $b=1$, and one end point called internal in the interior of $\mathbf{P}^{+}$or on the boundary $a+b=1:$ this segment is called a $K$-ligament.

- Any finite minimal kneading sequence terminating $C$ is realised on a single segment of straight line parallel to the $b$-axis, with one external end point on the line $a=1$, and one end point called internal in the interior of $\mathbf{P}^{+}$or on the boundary $a+b=1$ : this segment is called a $C$-ligament; we say ligament for either $K$-ligament or $C$-ligament.

- For any $n>1$ and a permutation $s$ on $n$ elements obtainable by restricting a $\{+-+\}$ bimodal map to one of its periodic orbits, the bone $B_{s}$ is the set of pairs $(a, b)$ such that, for $T_{a, b}^{+}$, one of the turning points belongs to a periodic orbit where the restriction of $T_{a, b}^{+}$acts like $s$. Any bone is made of two connected curves $B_{s}^{C}$ and $B_{s}^{K}$ where the superscript indicates which turning point belongs to the periodic orbit. The curves $B_{s}^{C}$ and $B_{s}^{K}$ intersect at two points, one of which is a $\chi$-point, the other one corresponding to the coexistence of two periodic orbits with the same $s$, each of which contains a turning point. The curve $B_{s}^{C}$ has two segments parallel to the $a$-axis, each having one external end point on the line $b=1$. The interior end points of these segments are joined by a piece of $K$-ligament which belongs to $B_{s}^{C}$. The curve $B_{s}^{K}$ has two segments parallel to the $b$-axis, each having with one external end point on the line $a=1$. The interior end points of these segments are joined by a piece of $C$-ligament which belongs to $B_{s}^{K}$.

Figures $1.1,1.2$, and 1.3 represent (not to scale) respectively $\alpha, \chi$, and $\psi$ points, the ligaments which cross at these points, and the ligaments and pieces of bones so generated in $\mathbf{P}^{+}$. These figures give a geometrical interpretation of Lemma D and of Theorems 1 and 2. Since some attention was paid in [MaT1MaT3] to the relations of a bone $B_{s}$ to the two bones corresponding to periodic orbits obtained from those in $B_{s}$ by a period-doubling bifurcation, Figure 1.4 shows how such triples of bones fit in the emanation discussion. In these figures, heavy black lines correspond to bones or to the lines $a=1$ and $b=1$; light black lines correspond to ligaments. The grey region in Figure 1.2 is the domain where the periodic orbit with maximal itinerary $\left(A K^{\rho(A)} B C^{-\rho(B)}\right)^{\infty}$ and minimal itinerary $\left(B D^{-\rho(B)} A K^{\rho(A)}\right)^{\infty}$ is an attractor. To simplify the annotation of the figures, we write $W Q^{+}$for $W Q^{\rho(W)}$ and $W Q^{-}$for $W Q^{-\rho(W)}$. In Figures 1.1, 1.2, and 1.3, if the grey lines are contracted to points (without creating further crossings on the black lines) one gets singular simplices which 


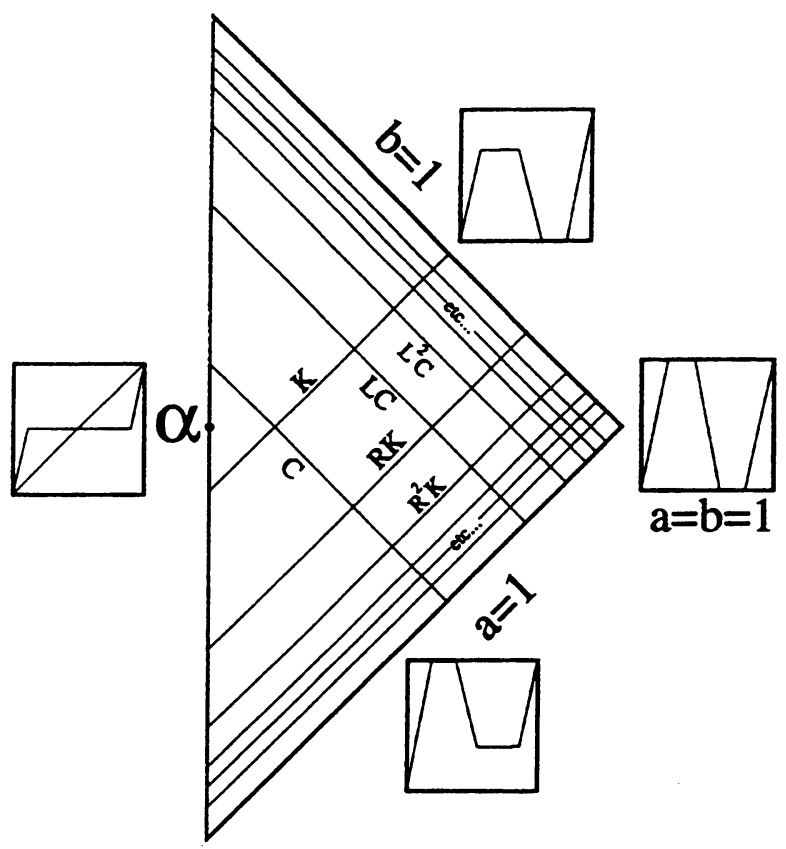

FIGURE 1.1. The $\alpha$-seed in the family $T_{a, b}^{+}$

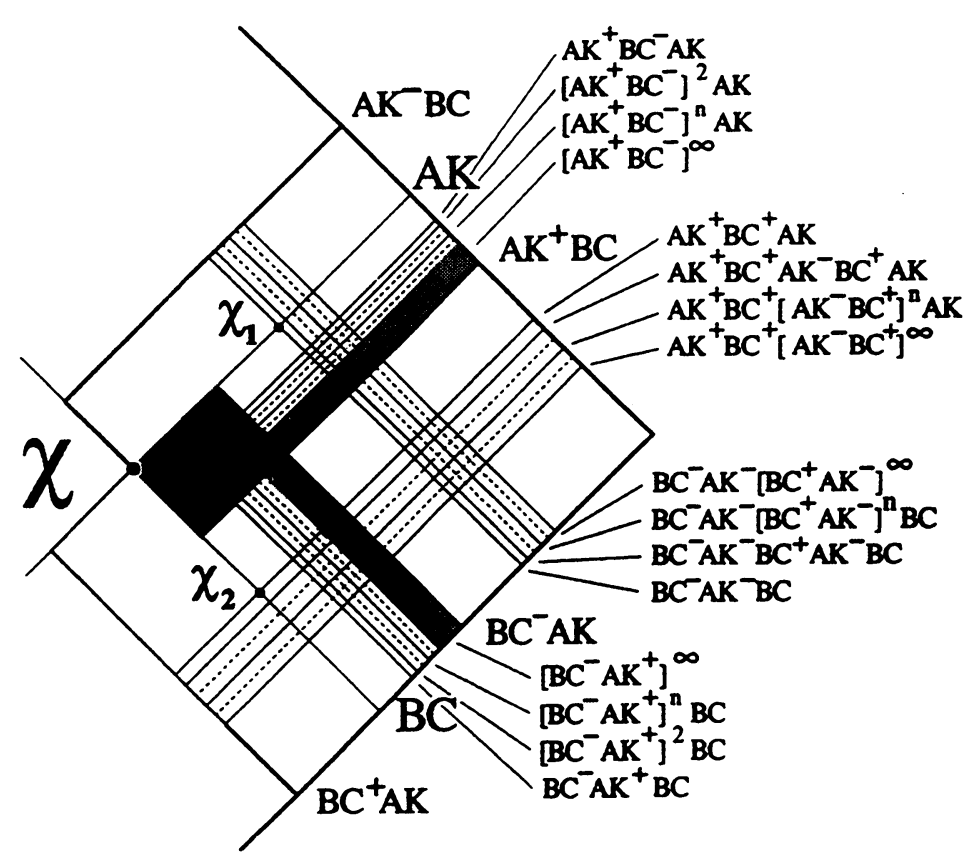

FIGURE 1.2. A $\chi$-seed in the family $T_{a, b}^{+}$ 


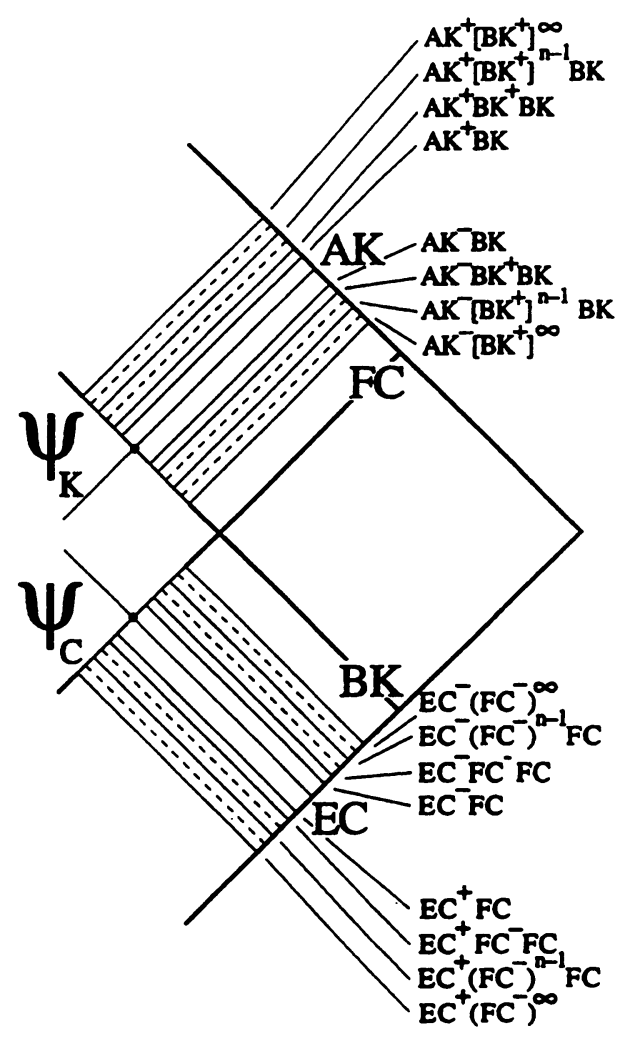

FIGURE 1.3. Two $\psi$-seeds in the family $T_{a, b}^{+}$

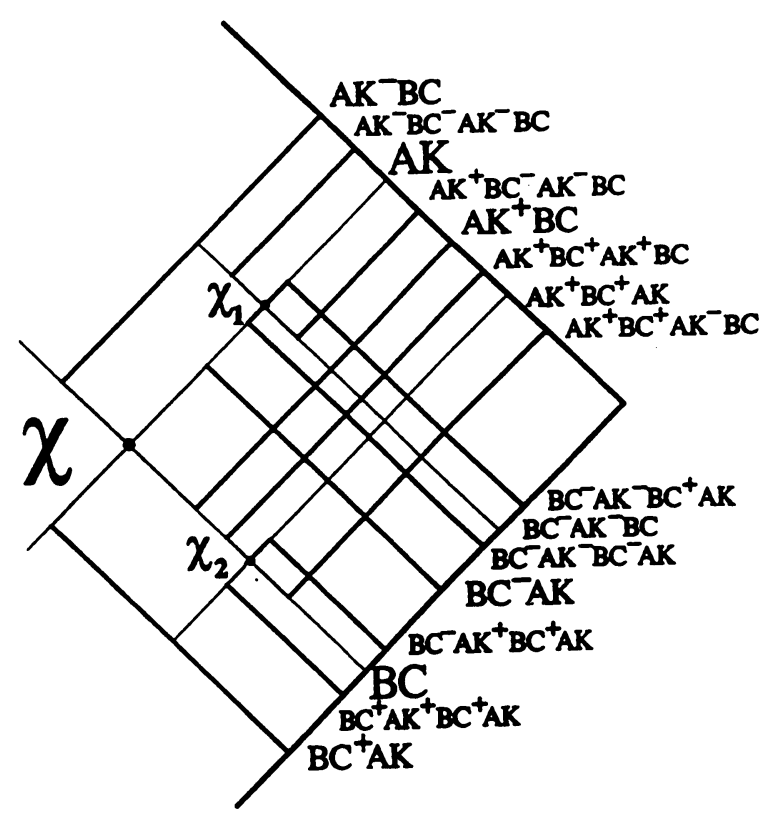

Figure 1.4. Period-doubling in the family $T_{a, b}^{+}$ 
are embedded in the plane as would conjecturally be the corresponding sets of bones and ligaments for a two-parameter family of cubic maps (see [DGMT]). In Figure 1.1, only the interior of the segment $a+b=1$ should be contracted to a point; in all cases, the contraction should take place without affecting the underlying bone. Figures $2.1-2.4$ are counterparts respectively to Figures $1.1-1.4$, obtained numerically from the cubic family given by $f_{a, b}(x)=x^{3}-a x+b$. Figure 0 showed for this family the region of its parameter space that corresponds most closely with $\mathbf{P}^{+}$: in the region shown in that figure, the maps are all bimodal except at the $\alpha$-seed $(a=b=0)$, and there is an invariant interval containing both critical points.

Proof of Theorem 3. Maximal kneading sequences are monotonically increasing with $a$, for fixed $b$, and with $b$, for fixed $a$. Similarly, minimal kneading sequences are monotonically decreasing with $a$, for fixed $b$, and with $b$, for fixed $a$. Consequently, the topological entropy of $T_{a, b}^{+}$increases when any of the parameters increases, the other parameter being fixed. Hence, the topological entropy on a ligament is minimal at its internal end point. On a bone, $B_{s}=B_{s}^{C} \cup B_{s}^{K}$, the topological entropy of $T_{a, b}^{+}$is minimal at the corners of $B_{s}^{C}$ and $B_{s}^{K}$ which are closest to the line $a+b=1$. Since up to a finite number of periodic orbits, the periodic orbit structure of $T_{a, b}^{+}$is unchanged on the ligament part of each bone (they correspond to lines in a hyperbolic region on the polynomial case), the topological entropy is unchanged there, and can only change by increasing when following a nonligament part parallel to either the $a$-axis or the $b$-axis. This shows that, at $\alpha, \chi$, and $\psi$ points, the topological entropy is not greater than on the lines emanating there, which is what we had to prove.

Combining Theorems 2 and 3 with the elementary properties of the bones and ligaments in $\mathbf{P}^{+}$yields the following result:

Theorem 4. In $\mathbf{P}^{+}$, any two seeds can be joined by finite path made of pieces from finitely many bones and ligaments. 


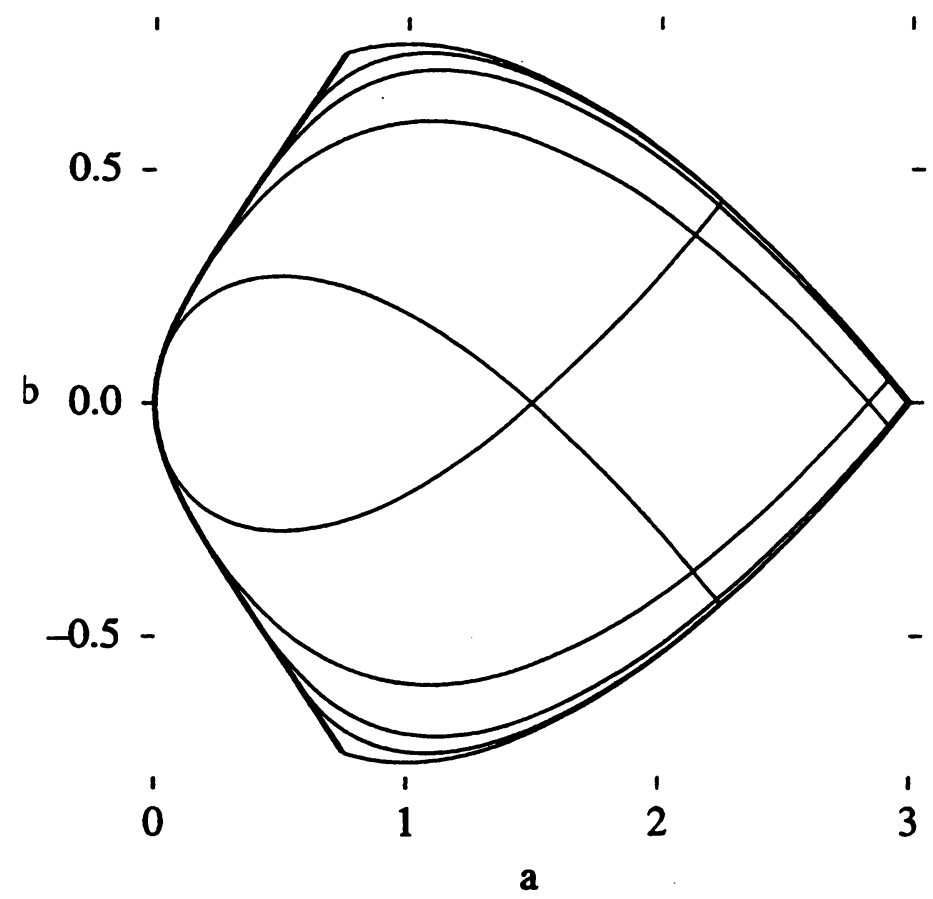

Figure 2.1. The $\alpha$-seed in the cubic family $f_{a, b}$

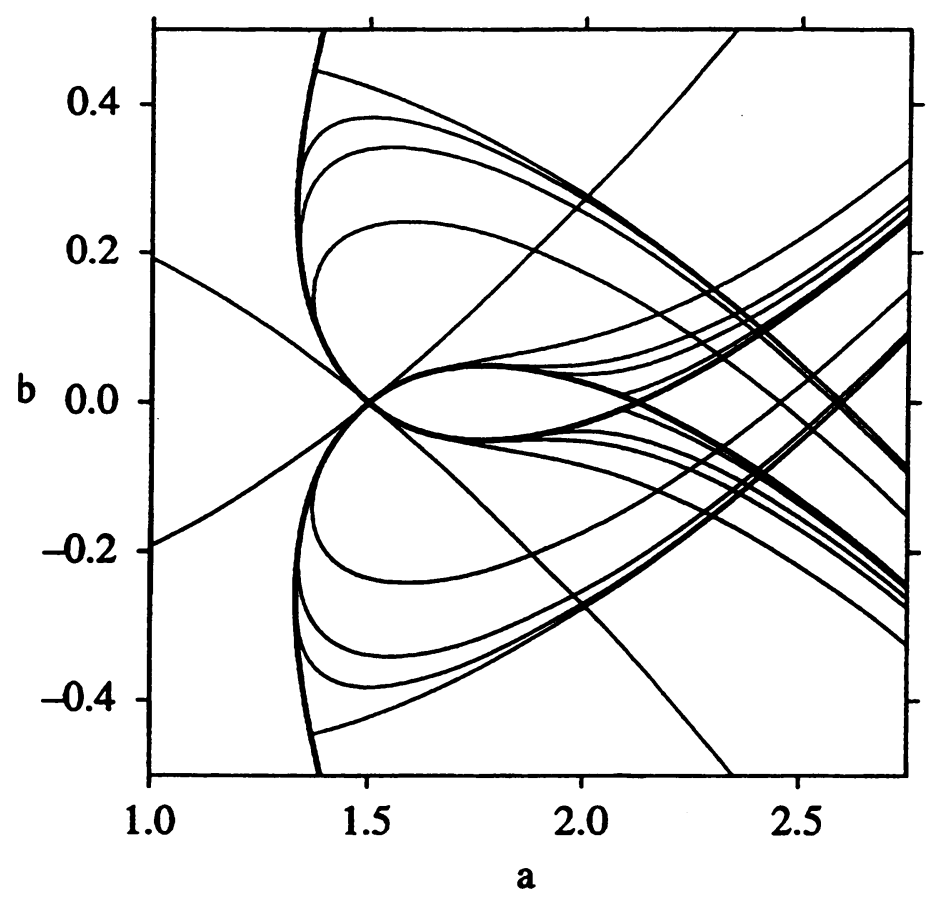

FIGURE 2.2. A $\chi$-seed in the family $f_{a, b}$ 


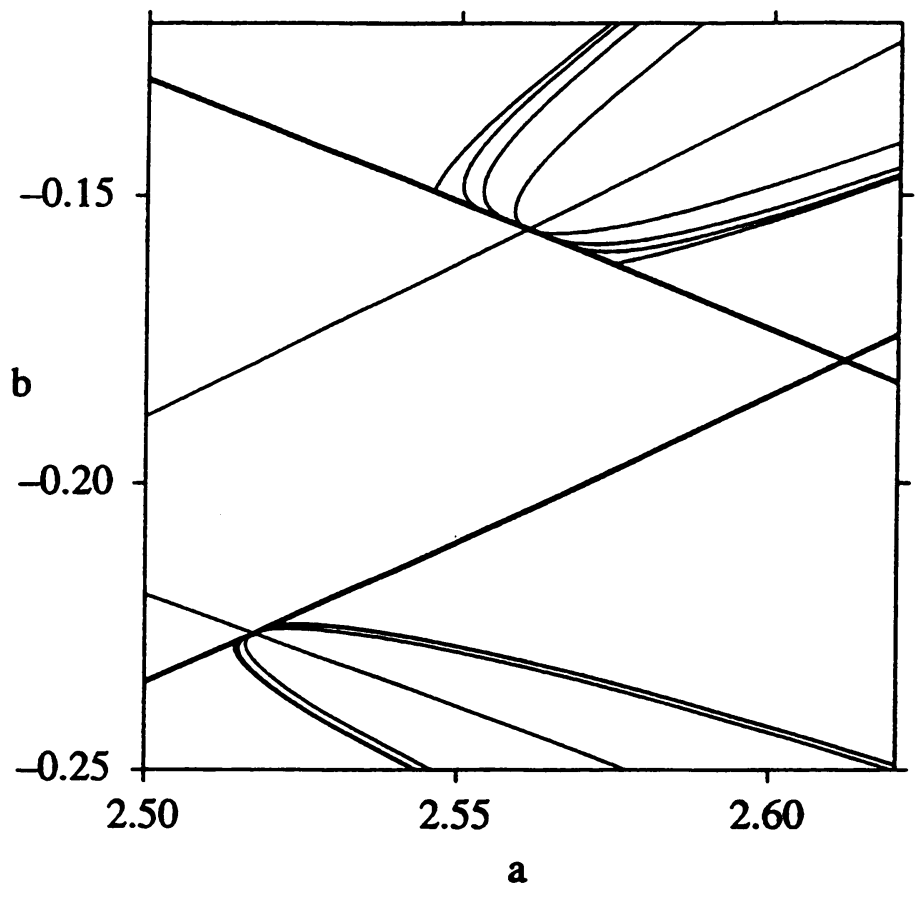

FIGURE 2.3. Two $\psi$-seeds in the family $f_{a, b}$

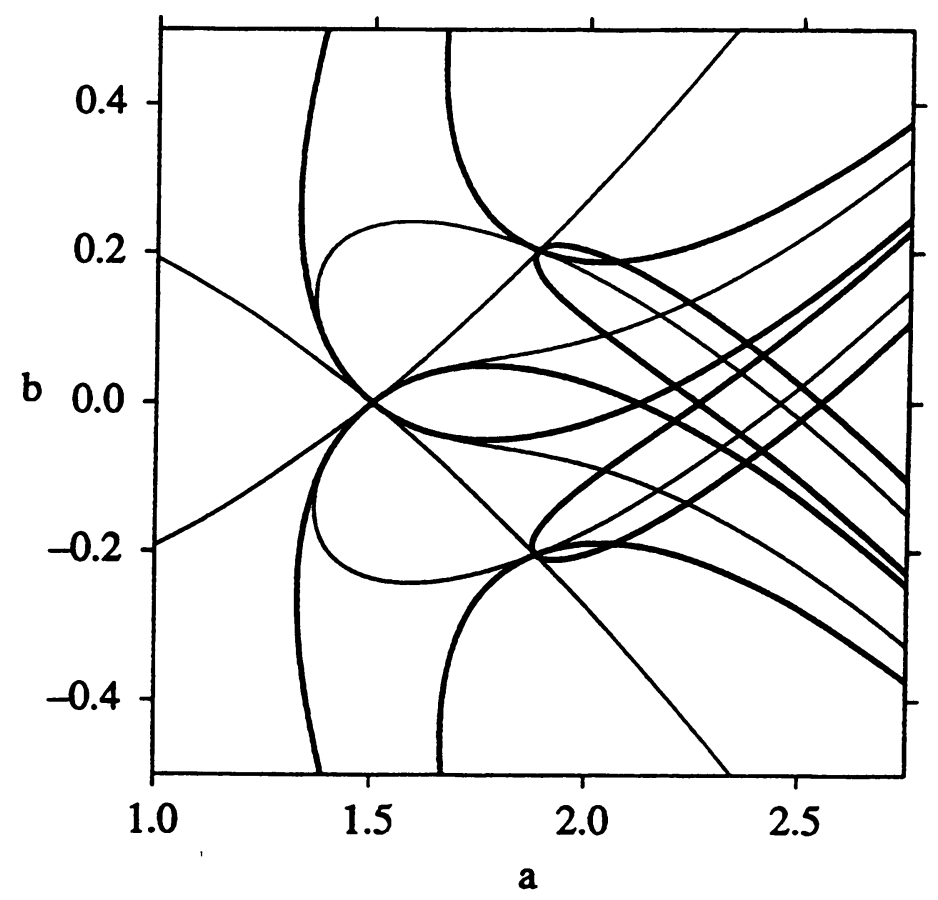

FIGURE 2.4. Period-doubling in the family $f_{a, b}$ 


\section{REFERENCES}

[B] P. L. Boyland, Bifurcations of circle maps: Arnol'd tongues, bistability and rotation intervals, Comm. Math. Phys. 106 (1986), 353-381.

[DGMT] S. P. Dawson, R. Galeeva, J. Milnor, and C. Tresser, Real and complex dynamical systems (B. Branner and P. Hjorth, eds.), Kluwer, Dordrecht, 1995.

[D] R. L. Devaney, Genealogy of periodic points of maps of the interval, Trans. Amer. Math. Soc. 265 (1981), 137-146.

[FK] S. Fraser and R. Kapral, Universal vector scaling in one-dimensional maps, Phys. Rev. A 30 (1984), 1017-1025.

[GT] J. M. Gambaudo and C. Tresser, $A$ monotonicity property in one dimensional dynamics, Contemporary Math., vol. 135, Amer. Math. Soc., Providence, RI, 1992, pp. 213-222.

[GP] L. Glass and R. Perez, Fine structure of phase locking, Phys. Rev. Lett. 48 (1982), 1772-1775.

[MaT1] R. S. Mackay and C. Tresser, Transition to topological chaos for circle maps, Physica 19D (1986), 206-237.

[MaT2] __ Some flesh on the skeleton: the bifurcation structure of bimodal maps, Physica 27D (1987), 412-422.

[MaT3] _ Boundary of topological chaos for bimodal maps of the interval, J. London Math. Soc. 37 (1988), 164-181.

[Mi1] J. Milnor, Remarks on iterated cubic maps, Experimental Math. 1 (1992), 5-24.

[MT] J. Milnor and W. Thurston, On iterated maps of the interval, Lecture Notes in Math., vol. 1342, Springer, 1988, pp. 465-563.

[M] P. Mumbrù and I. Rodriguez, Estructura, periodica i entropia topològica de les applications bimodals, Doctoral Thesis, Universidad Autónoma de Barcelona, 1987.

[R] J. Ringland, A genealogy for the periodic orbits of a class of 1D, Physica D 79 (1994), 289-298.

[RS1] J. Ringland and M. Schell, The Farey tree embodied-in bimodal maps of the interval, Phys. Lett. A 136 (1989), 379-386.

[RS2] _ Genealogy and bifurcation skeleton for cycles of the iterated two-extremum map of the interval, SIAM J. Math. Anal. 22 (1989), 1354-1371.

[STZ] R. Siegel, C. Tresser, and G. Zettler, A decoding problem in dynamics and in number theory, Chaos 2 (1992), 473-493.

[TW] C. Tresser and R. F. Williams, Splitting words and Lorenz braids, Physica D 62 (1993), 15-21.

See also W.-Z. Zeng and L. Glass, Symbolic dynamics and skeletons of circle maps, Physica D 40 (1989), 218-234.

Department of Mathematics, SUNY at Buffalo, Buffalo, New York 14214

E-mail address: ringlandeacsu. buffalo.edu

I.B.M., P.O. BOX 218, YoRKTOWN Heights, NEW YORK 10598 